\title{
Ordinal Bayesian Incentive Compatibility in Restricted Domains *
}

\author{
Debasis Mishra $^{\dagger}$
}

October 12, 2015

\begin{abstract}
We study deterministic voting mechanisms by considering an ordinal notion of Bayesian incentive compatibility (OBIC). If the beliefs of agents are independent and generic, we show that a mechanism is OBIC and satisfies an additional condition called elementary monotonicity if and only if it is a dominant strategy incentive compatible mechanism. Our result works in a large class of preference domains (that include the unrestricted domain, the single peaked domain, the single dipped domain, and some single crossing domains). We can significantly weaken elementary monotonicity in our result in the single peaked domain if we assume unanimity and in a large class of domains if we assume unanimity and tops-onlyness.
\end{abstract}

JEL Codes: D71, D82

Keywords: Ordinal Bayesian incentive compatibility, single peaked domain, elementary monotonicity

\footnotetext{
*An earlier version of this paper was titled "A Foundation for Dominant Strategy Voting Mechanisms". I am grateful to an associate editor and two anonymous referees for their extensive comments on an earlier version of the paper. I also thank Sarvesh Bandhu, Gabriel Carroll, Alexey Kushnir, Dipjyoti Majumdar, Benny Moldovanu, Herve Moulin, Anup Pramanik, Arunava Sen, and seminar participants at the Osaka University for helpful comments and suggestions. Parts of this research was carried out when I was visiting Institute of Social and Economic Research (ISER) at Osaka University. I am grateful for the hospitality and support provided by ISER, Osaka University.

${ }^{\dagger}$ Indian Statistical Institute, Delhi. Email: dmishra@isid.ac.in.
} 


\section{INTRODUCTION}

In standard models of voting, dominant strategy incentive compatibility (DSIC) is usually too demanding. This is illustrated by the Gibbard-Satterthwaite theorem (Gibbard, 1973; Satterthwaite, 1975), which shows that the only DSIC and unanimous deterministic voting mechanism in the unrestricted domain is a dictatorship. ${ }^{1}$ This motivates the study of weaker solution concepts in these models. In this paper, we consider ordinal Bayesian incentive compatibility (OBIC) introduced by d'Aspremont and Peleg (1988). A voting mechanism is OBIC if for every agent, his interim/expected outcome probability vector from truth-telling first-order stochastic-dominates any interim outcome probability vector obtained by deviating. In the unrestricted domain of preferences, Majumdar and Sen (2004) show that OBIC with independent and generic priors is equivalent to DSIC under unanimity. ${ }^{2}$ We investigate the robustness of this result to the unrestricted domain assumption.

We construct a non-DSIC, unanimous, and anonymous mechanism that is OBIC with respect to some generic priors when the domain of preferences is restricted to be the single peaked domain. However, our main results suggest that the equivalence between OBIC and DSIC voting mechanisms can be restored in various restricted domains under weak additional axioms. The main additional axioms that we use are elementary monotonicity and its weaker versions along with unanimity. Elementary monotonicity, which we formally define later, is a very mild form of Maskin monotonicity, and requires a mechanism to respond positively to changes in the preferences of agents. It is satisfied by a variety of mechanisms.

Our core result says that OBIC and elementary monotonicity are equivalent to DSIC in a large class of domains. In the single peaked domain, the equivalence between OBIC and DSIC holds with a significantly weaker version of elementary monotonicity if we assume unanimity. If we assume unanimity and tops-onlyness, the weakened version of elementary monotonicity and OBIC are equivalent to DSIC in a large class of domains.

Our results provide a foundation for using dominant strategy voting mechanisms in various restricted domains. An implication of our results is that if we want to design Bayesian incentive compatible voting mechanisms, we must consider randomized and/or cardinal mechanisms. All our results hold even if we weaken OBIC to only prevent manipulations of each agent to his adjacent preferences - we call this requirement locally OBIC (LOBIC). Incentive compatibility with local incentive constraints were recently studied in Carroll (2012) and

\footnotetext{
${ }^{1}$ Throughout the paper, we only consider deterministic voting mechanisms.

${ }^{2}$ We define a generic prior formally later - it is a generic subset of the set of independent priors.
} 
Sato (2013), who identified domains where local incentive constraints imply all incentive constraints. All our proofs crucially use ideas from this literature. Thus, our results bring together two different ideas (OBIC and local incentive compatibility) in strategic voting literature.

Our results extend the result in Majumdar and Sen (2004) by identifying the precise connection between DSIC and OBIC mechanisms with (and without) unanimity in restricted domains. Our results corroborate the different implications of OBIC with generic and uniform priors (a uniform prior requires that each preference in the domain is drawn with equal probability). This is because Majumdar and Sen (2004) had shown that every neutral mechanism satisfying elementary monotonicity is OBIC under uniform priors in the unrestricted domain - this covers many reasonable mechanisms. In contrast, our results show a very different implication of elementary monotonicity with generic priors.

\subsection{Relation to Literature}

Our results parallel recent contributions in the single object auction quasilinear utility models (and some of its extensions) by Manelli and Vincent (2010) and Gershkov et al. (2013), who establish a weaker version of equivalence between Bayesian incentive compatible and DSIC mechanisms. The equivalence in these papers is in terms of interim outcome probabilities. Besides, these papers look at cardinal mechanisms with quasilinearity, whereas ours is a completely ordinal environment without transfer.

One way to interpret our results is that we are replacing DSIC by weaker axioms - LO$\mathrm{BIC}$ and some additional axioms. In a recent paper, such an approach of decomposing the DSIC axiom is pursued in Muto and Sato (2014). They use three weaker axioms than DSIC and show that they are equivalent to DSIC in the unrestricted domain. Though they do not consider OBIC, some of their axioms (more precisely, some weakening of their axioms) are implied by OBIC with generic priors. Their result only applies to the unrestricted domains, while our results apply to restricted domains.

Besides Majumdar and Sen (2004), Bhargava et al. (2014) study OBIC voting mechanisms with correlated priors in the unrestricted domain. They show that certain types of correlation allows one to escape the Gibbard-Satterthwaite impossibility result. OBIC mechanisms have been studied in the context of matching problems in Majumdar (2003); Ehlers and Masso $(2007,2015)$. These papers study the implication of stability and OBIC in two-sided matching problems. Though some of our results extend to private good allocation 
problems, most of our results are specific to voting environment.

The rest of the paper is organized as follows. Section 2 introduces the model. Section 3 formally states the unrestricted domain result and gives two counterexamples to show that the result breaks down in a restricted domain or if the number alternatives is two. Section 4 formally defines all the domains we use in the paper. Section 5 contains all the results and Section 5.5 gives specific examples of domains where our results can be applied. We end with some discussions in Section 6.

\section{The Model}

We formally introduce our model in this section. Let $A$ be a finite set of alternatives and $\mathcal{P}$ be the set of all strict linear orders over $A$. Let $\mathcal{D} \subseteq \mathcal{P}$ be some subset of strict linear orders. We will refer to $\mathcal{D}$ as the domain. There are $n$ agents. The set of agents is denoted by $N=\{1, \ldots, n\}$. The private preference/type of each agent $i \in N$ is a strict linear order $P_{i} \in \mathcal{D}$.

A social choice function (scf) is a map $f: \mathcal{D}^{n} \rightarrow A .^{3}$

Definition 1 An scf $f: \mathcal{D}^{n} \rightarrow A$ is dominant strategy incentive compatible (DSIC) if for every $i \in N$, every $P_{-i}$, and every $P_{i} \in \mathcal{D}$, there exists no $P_{i}^{\prime} \in \mathcal{D}$ such that

$$
f\left(P_{i}^{\prime}, P_{-i}\right) P_{i} f\left(P_{i}, P_{-i}\right) .
$$

To introduce the notion of ordinal Bayesian incentive compatibility, we first define assumption that we make about the priors in our model. We will assume that there are common beliefs that each agent $i$ independently draws his preference using a probability distribution $\mu_{i}: \mathcal{D} \rightarrow[0,1]$. Hence, the belief of agent $i$ that agents other than $i$ have a preference profile $P_{-i}$ will be denoted as $\mu\left(P_{-i}\right) \equiv \times_{j \neq i} \mu_{j}\left(P_{j}\right)$. We will refer to $\left\{\mu_{i}\right\}_{i \in N}$ as a profile of priors.

We make the following assumption about generic priors in the paper.

Definition 2 The profile of priors $\left\{\mu_{i}\right\}_{i \in N}$ is generic if for every $j \in N$ and for every $\mathcal{S}, \mathcal{T} \subseteq \mathcal{D}^{n-1}$ we have

$$
\left[\sum_{P_{-j} \in \mathcal{S}} \mu\left(P_{-j}\right)=\sum_{P_{-j} \in \mathcal{T}} \mu\left(P_{-j}\right)\right] \Rightarrow[\mathcal{S}=\mathcal{T}] .
$$

\footnotetext{
${ }^{3} \mathrm{An}$ scf is a direct revelation mechanism, and by the revelation principle, it is without loss of generality to focus attention on such mechanisms.
} 
Genericity requires that if we consider an agent $j$ and consider two distinct subsets of profiles of preferences of agents in $N \backslash\{j\}$, then the probability that agents in $N \backslash\{j\}$ have preferences in these subsets cannot be the same. Mathematically, these priors are generic in a topological sense (Majumdar and Sen, 2004) - to be precise, Majumdar and Sen (2004) show this fact when $\mathcal{D}=\mathcal{P}$, but an identical proof works if $\mathcal{D} \subseteq \mathcal{P}$. ${ }^{4}$ One notable prior that is not generic is the uniform prior that assigns the same probability to all the preferences.

Given a social choice function $f$, we can compute the interim outcome probability of each agent from this scf using the priors. For this, consider a profile of priors $\left\{\mu_{i}\right\}_{i \in N}$. For each agent $i \in N$, define $\pi_{i}^{f}\left(a, P_{i}\right)$ as the interim outcome probability of the scf $f$ choosing alternative $a$ when agent $i$ reports $P_{i}$ as his preference (and other agents report truthfully):

$$
\pi_{i}^{f}\left(a, P_{i}\right)=\sum_{P_{-i} \in \mathcal{D}^{n-1}: f\left(P_{i}, P_{-i}\right)=a} \mu\left(P_{-i}\right) .
$$

Note that $\pi_{i}^{f}$ depends on the priors, but we have suppressed it to make the notation less complex.

For any alternative $a \in A$ and any $P_{i} \in \mathcal{D}$, let $B\left(a, P_{i}\right):=\{a\} \cup\left\{b \in A: b P_{i} a\right\}$.

Definition 3 (d'Aspremont and Peleg (1988)) An scf $f$ is ordinally Bayesian incentive compatible (OBIC) with respect to profile of priors $\left\{\mu_{i}\right\}_{i \in N}$ if for every $i \in N$, for every $P_{i}, P_{i}^{\prime} \in \mathcal{D}$, and for every $a \in A$, we have

$$
\sum_{b \in B\left(a, P_{i}\right)} \pi_{i}^{f}\left(b, P_{i}\right) \geq \sum_{b \in B\left(a, P_{i}\right)} \pi_{i}^{f}\left(b, P_{i}^{\prime}\right) .
$$

An equivalent definition of $\mathrm{OBIC}$ is to require that for every $i \in N$, for every $P_{i}, P_{i}^{\prime} \in \mathcal{D}$, for every utility function $u: A \rightarrow \mathbb{R}$ representing $P_{i}$, we have

$$
\sum_{a \in A} u(a) \pi_{i}^{f}\left(a, P_{i}\right) \geq \sum_{a \in A} u(a) \pi_{i}^{f}\left(a, P_{i}^{\prime}\right) .
$$

A well known fact to note is that if an scf is OBIC with respect to all beliefs, then it is DSIC.

Definition 4 An scf $f$ is $\mathbf{G - O B I C}$ if there exists a profile of generic priors $\left\{\mu_{i}\right\}_{i \in N}$ such that $f$ is $O B I C$ with respect to $\left\{\mu_{i}\right\}_{i \in N}$.

G-OBIC requires OBIC with respect to some profile of generic priors (it may be just one profile of generic priors), but need not be all profile of generic priors.

\footnotetext{
${ }^{4}$ Denote by $\Delta$ the unit simplex of dimension $|\mathcal{D}|-1$. The set of common independent beliefs is the $n$-th order Cartesian product of unit simplices $\Delta$, and is given by $\Delta^{n}$. The set of priors ruled out by genericity are given by equations that define a finite set of hyperplanes in $\Delta^{n}$, which has Lebesgue measure zero in $\Delta^{n}$.
} 


\section{The Unrestricted Domain Result}

We now discuss the implication of G-OBIC along with unanimity in the unrestricted domain. At any preference ordering $P$, we will denote by $P(k)$ the $k$-th ranked alternative according to $P$.

Definition 5 An scf $f: \mathcal{D}^{n} \rightarrow A$ is unanimous if for every $\mathbf{P} \equiv\left(P_{1}, \ldots, P_{n}\right)$ with $P_{1}(1)=\ldots=P_{n}(1)$, we have $f(\mathbf{P})=P_{1}(1)$.

The following result extends the Gibbard-Satterthwaite theorem using G-OBIC in the unrestricted domain.

TheOREM 1 (Majumdar and Sen (2004)) Let $|A| \geq 3$ and $f: \mathcal{P}^{n} \rightarrow A$ be a unanimous scf, where $\mathcal{P}$ is the unrestricted domain. Then, $f$ is G-OBIC if and only if it is DSIC.

The proof of this result in Majumdar and Sen (2004) is direct. Instead of showing that every unanimous and G-OBIC scf is DSIC, and then using the Gibbard-Satterthwaite theorem to conclude dictatorship, they directly prove that G-OBIC and unanimity imply dictatorship. Hence, one does not obtain any intuition from their proof whether Theorem 1 will hold in other domains.

\subsection{A Two Alternatives Example}

We give an example with two alternatives to show how Theorem 1 can break down. The example gives insights on why G-OBIC and non-DSIC mechanism may exist when the assumptions of Theorem 1 are relaxed.

Let $A=\{a, b\}$ and $N=\{1,2,3\}$. We define an scf $\bar{f}$ as follows. If all the agents have the same top ranked alternative, then $\bar{f}$ picks that alternative. Else, $\bar{f}$ picks the alternative which is top ranked for less number of agents.

Clearly, $\bar{f}$ is unanimous. We next argue that $\bar{f}$ is G-OBIC. Since there are only two alternatives, we only need to show that $\pi_{i}^{\bar{f}}\left(P_{i}(1), P_{i}\right) \geq \pi_{i}^{\bar{f}}\left(P_{i}(1), P_{i}^{\prime}\right)$ for all $i \in N$, for all $P_{i}, P_{i}^{\prime}$. For simplicity, for every agent $i \in N$, we will denote the preference ordering where $a$ is top ranked as $P_{i}$ and the preference ordering where $b$ is top ranked as $P_{i}^{\prime}$. For every $i \in N$, let the probability that agent $i$ has type $P_{i}$ be $p_{i}$ and the probability that he has type $P_{i}^{\prime}$ be $\left(1-p_{i}\right)$. Now, we can compute the interim allocation probabilities for agent 1 as follows. 
If agent 1 has type $P_{1}$ (where $a$ is top ranked), then the probability that $b$ will be the outcome in $\bar{f}$ is the probability that agents 2 and 3 have different types. This probability is exactly $p_{2}\left(1-p_{3}\right)+p_{3}\left(1-p_{2}\right)=p_{2}+p_{3}-2 p_{2} p_{3}$. Hence, if agent 1 has type $P_{1}$, then the probability that $a$ will be the outcome in $\bar{f}$ is $1-p_{2}-p_{3}+2 p_{2} p_{3}$. An analogous calculation can be used to compute interim outcome probabilities when agent 1 has type $P_{1}^{\prime}$. This is summarized in Table 1.

\begin{tabular}{|c|c|c|}
\hline & $P_{1}$ & $P_{1}^{\prime}$ \\
$\pi_{1}^{\bar{f}}(a, \cdot)$ & $1-p_{2}-p_{3}+2 p_{2} p_{3}$ & $p_{2}+p_{3}-2 p_{2} p_{3}$ \\
$\pi_{1}^{\bar{f}}(b, \cdot)$ & $p_{2}+p_{3}-2 p_{2} p_{3}$ & $1-p_{2}-p_{3}+2 p_{2} p_{3}$ \\
\hline
\end{tabular}

Table 1: Interim outcome probabilities of agent 1 in $\bar{f}$

The interim outcome probabilities of agents 2 and 3 can be computed in an analogous manner. It is easily seen from Table 1 that OBIC constraints can be satisfied for agent 1 if and only if $1-p_{2}-p_{3}+2 p_{2} p_{3} \geq p_{2}+p_{3}-2 p_{2} p_{3}$, which is equivalent to requiring that $\left(1-2 p_{2}\right)\left(1-2 p_{3}\right) \geq 0$.

Collecting the OBIC constraints for all agents, we can then conclude that $\bar{f}$ is OBIC if and only if priors satisfy

$$
\begin{aligned}
& \left(1-2 p_{2}\right)\left(1-2 p_{3}\right) \geq 0 \\
& \left(1-2 p_{1}\right)\left(1-2 p_{3}\right) \geq 0 \\
& \left(1-2 p_{1}\right)\left(1-2 p_{2}\right) \geq 0
\end{aligned}
$$

This is possible if and only if either $p_{1}, p_{2}, p_{3} \in(0,0.5)$ or $p_{1}, p_{2}, p_{3} \in(0.5,1)$. To see why $p_{1}, p_{2}, p_{3}$ can be picked such that the priors become generic, note that the set of priors satisfying either $p_{1}, p_{2}, p_{3} \in(0,0.5)$ or $p_{1}, p_{2}, p_{3} \in(0.5,1)$ is a subset of $(0,1)^{3}$ with a non-empty interior. Hence, it will have a non-empty intersection with the set of generic priors. ${ }^{5}$

Intuitively, if agent 1 has $a$ as top, then the only profile where agent 1 gets $a$ as outcome are when the other two agents have the same preference. So, if the probability that any pair of agents have the same preference is high enough, then interim outcome probability of $a$ will be high, and the mechanism will be OBIC. This is leading to the conditions we have derived.

\footnotetext{
${ }^{5}$ For instance, one can verify that $p_{1}=0.49, p_{2}=0.47, p_{3}=0.43$ results in generic priors.
} 


\subsection{An Example in the Single Peaked Domain}

We now extend the two alternatives example to the single peaked domain. Single peaked domain is an important domain restriction in strategic voting literature with applications in political economy and other disciplines. It is a domain where existence of anonymous, unanimous, and DSIC scfs is guaranteed (Moulin, 1980), and thus, allows one to escape the negative consequences of the Gibbard-Satterthwaite theorem. ${ }^{6}$ In this domain, we give an example of an scf with three alternatives and three agents that is G-OBIC, unanimous, and anonymous but not DSIC. ${ }^{7}$

The single peaked domain is defined as follows. Let $\succ$ be a strict linear order of the set of alternatives $A$.

Definition 6 A preference ordering $P$ is single peaked with respect to $\succ$ if for every $b, c \in A$ with $P(1) \succ b \succ c$ or $c \succ b \succ P(1)$, we have $b P c$.

We assume that $A=\{a, b, c\}$ and $N=\{1,2,3\}$. Suppose the preferences are single peaked with respect to the strict linear order $\succ$ given by $a \succ b \succ c$. The set of all single peaked preference orderings with respect to $\succ$ is denoted by $\mathcal{S}$. For this example, the domain $\mathcal{S}$ is shown in Table 2, where each column is a preference in $\mathcal{S}$.

$$
\begin{array}{l|l|l|l}
a & b & b & c \\
b & a & c & b \\
c & c & a & a
\end{array}
$$

Table 2: The single peaked domain

Our scf considers the number of agents who prefer $a$ to $b$ and the number of agents who prefer $b$ to $a$. We say $a$ is a loser in $\{a, b\}$ at $\mathbf{P}$ if $\left|\left\{i \in N: a P_{i} b\right\}\right|<\left|\left\{i \in N: b P_{i} a\right\}\right|$. Else, we say $b$ is a loser in $\{a, b\}$ at $\mathbf{P}$. Now, the scf $f^{*}$ is defined as follows. For any preference profile $\mathbf{P} \in \mathcal{S}^{3}$,

1. if all the agents have the same top ranked alternative then $f^{*}$ chooses that alternative,

2. if all the agents do not have the same top ranked alternative but every agent prefers $b$ to $a$, then $f^{*}(\mathbf{P})=b$,

\footnotetext{
${ }^{6}$ Conversely, Chatterji et al. (2013) have shown that any domain that admits a "well-behaved" social choice function must be roughly single peaked.

${ }^{7}$ Informally, anonymity requires that if we permute the preferences of agents and consider the new profile of preferences, the outcome at the new profile must be the same as the old one.
} 
3. if both the above conditions fail, then $f^{*}$ chooses the loser alternative at $\mathbf{P}$.

The following proposition shows that $f^{*}$ is G-OBIC.

Proposition 1 The scf $f^{*}$ is unanimous, anonymous, and G-OBIC, but not DSIC.

The proof of Proposition 1 and all subsequent proofs are given in the Appendix. The intuition for why Proposition 1 works is similar to that of the two alternatives example. In contrast to the unrestricted domain, the set of preferences in the single peaked domain is less. This allows us to extend the two alternatives example in a natural way to this domain. However, in the unrestricted domain, there are too many manipulations to take care of, and these rules fail to be G-LOBIC.

\section{The Local Domains}

We will now formally define the restricted domains that we investigate in this paper. For each of these restricted domains, we show equivalence of G-OBIC and DSIC under some additional conditions.

All our results work in a class of restricted domains that we call local domains. To define these restricted domains, we first introduce the notion of local incentive compatibility. Consider an agent $i$ and two alternatives $a, b \in A$. Suppose $P_{i}$ is a preference ordering such that $P_{i}(k)=a$ and $P_{i}(k+1)=b$. Now, consider $P_{i}^{\prime}$ such that $P_{i}^{\prime}(k+1)=a, P_{i}^{\prime}(k)=b$, and $P_{i}^{\prime}(j)=P_{i}(j)$ for all $j \notin\{k, k+1\}$. In other words, $a$ and $b$ are consecutively ranked in $P_{i}$, and $P_{i}^{\prime}$ is constructed by swapping only their positions. In this case, we say that $P_{i}^{\prime}$ is a $(a, b)$-swap of $P_{i}$. Note that if $P_{i}^{\prime}$ is an $(a, b)$-swap of $P_{i}$, then the position of $b$ improves from $P_{i}$ to $P_{i}^{\prime}$. Hence, $(a, b)$-swap is different from $(b, a)$-swap.

DEFINITION 7 An scf $f$ is locally dominant strategy incentive compatible (LDSIC) if for every $i \in N$, every $P_{-i} \in \mathcal{D}^{n-1}$, and every $P_{i} \in \mathcal{D}$ there exists no $P_{i}^{\prime} \in \mathcal{D}$ such that $P_{i}^{\prime}$ is an $(a, b)$-swap of $P_{i}$ for some $a, b \in A$ and

$$
f\left(P_{i}^{\prime}, P_{-i}\right) P_{i} f\left(P_{i}, P_{-i}\right) .
$$

Local DSIC only prevents manipulations across preferences which are swaps of each other. Using the notion of LDSIC, we now define a class of domains.

DeFinition 8 A domain $\mathcal{D}$ is a local domain if every LDSIC $f: \mathcal{D}^{n} \rightarrow A$ in that domain is also DSIC. 
Many interesting domains like the unrestricted domain, the single peaked domain, and a successive single crossing domain are known to be local domains (Carroll, 2012; Sato, 2013). We formally define some of these domains in Section 5.5.

We now introduce a subclass of local domains where we have stronger results. These are a class of local domains introduced in Sato (2013). Two preferences $P$ and $P^{\prime}$ are adjacent if there is $x, y \in A$ such that $P^{\prime}$ is an $(x, y)$-swap of $P$.

Definition $9 A$ distinct sequence of preferences $\left(P^{0}, P^{1}, \ldots, P^{k}, P^{k+1}\right)$ in $\mathcal{D}$ is without restoration if

- for every $j \in\{0,1, \ldots, k\}, P^{j}$ and $P^{j+1}$ are adjacent,

- there exists no distinct $j, j^{\prime} \in\{0,1, \ldots, k\}$ and $x, y \in A$ such that $P^{j+1}$ is a $(x, y)$-swap of $P^{j}$ and $P^{j^{\prime}+1}$ is a $(y, x)$-swap of $P^{j^{\prime}}$.

$A$ domain $\mathcal{D}$ is connected without restoration if for every $P, P^{\prime} \in \mathcal{D}$, there exists a sequence of distinct preferences $\left(P=P^{0}, P^{1}, \ldots, P^{k}, P^{k+1}=P^{\prime}\right)$ in $\mathcal{D}$ without restoration.

The without restoration property requires that no pair of alternatives is swapped more than once along the sequence. Sato (2013) shows that if a domain is connected without restoration, then it is a local domain. Further, he shows that the unrestricted domain and the single peaked domain are connected without restoration.

We remark that besides the single peaked domain, there are other interesting domains that are connected without restoration - see Sato (2013). We identify some specific domains that are connected without restoration in Section 5.5.

For some of our results, we will impose the following richness condition.

Definition 10 A domain $\mathcal{D}$ is rich if for every alternative a, there exists a preference ordering $P \in \mathcal{D}$ such that $P(1)=a$.

\section{The Results}

We will now present our results. Our results extend Theorem 1 to various restricted domains we have discussed under additional conditions. We define and discuss the additional conditions before stating the results. Though we use additional axioms, we use the following weaker notion of incentive compatibility than G-OBIC. 
DEFInITION 11 An scf $f$ is locally ordinally Bayesian incentive compatible (LOBIC) with respect to profile of priors $\left\{\mu_{i}\right\}_{i \in N}$ if for every $i \in N$, for every $P_{i}, P_{i}^{\prime} \in \mathcal{D}$ such that $P_{i}^{\prime}$ and $P_{i}$ are adjacent, and for every $a \in A$, we have

$$
\sum_{b \in B\left(a, P_{i}\right)} \pi_{i}^{f}\left(b, P_{i}\right) \geq \sum_{b \in B\left(a, P_{i}\right)} \pi_{i}^{f}\left(b, P_{i}^{\prime}\right)
$$

An scf $f$ is $\mathbf{G - L O B I C}$ if there exists some profile of generic priors $\left\{\mu_{i}\right\}_{i \in N}$ such that $f$ is $L O B I C$ with respect to $\left\{\mu_{i}\right\}_{i \in N}$.

Clearly, if $f$ is OBIC, then it is also LOBIC. In general, LOBIC is a very weak incentive compatibility requirement since it requires only a small subset of incentive constraints to hold. Further, LOBIC may not imply OBIC even in the local domain - local domain only requires that LDSIC implies DSIC.

\subsection{Equivalence in Local Domains}

Our first result uses one of the following two axioms.

Definition 12 An scf $f$ satisfies elementary monotonicity if for every $i \in N$, every $P_{-i} \in \mathcal{D}^{n-1}$, and every $P_{i}, P_{i}^{\prime} \in \mathcal{D}$ such that $P_{i}^{\prime}$ is a $(a, b)$-swap of $P_{i}$ for some $a, b \in A$ and $f\left(P_{i}, P_{-i}\right) \neq a$, we have $f\left(P_{i}^{\prime}, P_{-i}\right) \neq a$.

The next axiom is similar in spirit to elementary monotonicity.

DEFINITION 13 An scf $f$ is positively responsive if for every agent $i \in N$, for every preference profile $P_{-i}$, and for every $P_{i}, P_{i}^{\prime}$, with $f\left(P_{i}, P_{-i}\right)=a$ and $P_{i}^{\prime}(1)=a$, we have $f\left(P_{i}^{\prime}, P_{-i}\right)=a$.

Both the axioms are one-agent axioms - we fix preferences of other agents at some $P_{-i}$ and change the preference of agent $i$ from $P_{i}$ to $P_{i}^{\prime}$. These axioms require that the scf responds in a positive manner if the change in preferences lifts the outcome. In case of elementary monotonicity, the outcome is lifted from $P_{i}$ to $P_{i}^{\prime}$ by one position in a local way. In case of positive responsiveness, the outcome is lifted to the top in $P_{i}^{\prime}$, but the ranking among other alternatives are allowed to change. In both the cases, agent $i$ is providing positive support to the current outcome by lifting its position. In case of positive responsiveness, the support is more natural because it is lifted to the top.

These are relatively weak axioms and satisfied by many well know social choice functions. We refer to discussions on them in Moulin (1983); Majumdar and Sen (2004). We provide a brief discussion on them in Section 6. We are now ready to state our first main result. 
TheOREm 2 Let $f: \mathcal{D}^{n} \rightarrow A$ be an scf. Consider the following statements

1. $f$ is DSIC.

2. $f$ is $G$-LOBIC and elementary monotone.

3. $f$ is $G$-LOBIC and positively responsive.

Statements (1) and (2) are equivalent if $\mathcal{D}$ is a local domain. Statements (1), (2), and (3) are equivalent if $\mathcal{D}$ is a rich connected domain without restoration.

Note that Theorem 2 does not assume unanimity. It also does not require any condition on the range of the scf. An immediate corollary of Theorem 2 gives an indirect characterization of local domains.

Corollary 1 Suppose $\mathcal{D}$ is any domain. Every scf on $\mathcal{D}^{n}$ satisfying elementary monotonicity and $G$-LOBIC is DSIC if and only if $\mathcal{D}$ is a local domain.

Proof: Theorem 2 already establishes one direction. For the other direction, fix a domain $\mathcal{D}$ where every scf satisfying elementary monotonicity and G-LOBIC is DSIC. By Lemma 5 every LDSIC scf satisfies elementary monotonicity and G-LOBIC. So, every LDSIC scf is also DSIC. Hence, the domain is a local domain.

Another corollary of Theorem 2 is about the $n=2$ agents case. We show that Theorem 1 extends to $n=2$ agents case (independent of number of alternatives) in every rich connected domain without restoration.

Corollary 2 Suppose $\mathcal{D}$ is a rich connected domain without restoration and $n=2$. Let $f: \mathcal{D}^{n} \rightarrow A$ be a unanimous scf. Then, $f$ is DSIC if and only if it is G-LOBIC.

Corollary 2 explains why the scf in Proposition 1 had three agents.

\subsection{Equivalence in Single Peaked Domain}

We will now investigate subdomains where unanimity allows us to weaken elementary monotonicity (or positive responsiveness). Our strongest result comes in the single peaked domain, where we weaken elementary monotonicity as follows.

Fix a domain $\mathcal{D}$. A profile of preferences $\mathbf{P} \in \mathcal{D}^{n}$ is a top-2 profile if for every $i, j \in N$, $P_{i}(k)=P_{j}(k)$ for all $k>2$. At a top-2 profile, agents differ in their ranking of alternatives only for the top two alternatives. Further, if $\left(P_{i}, P_{-i}\right)$ and $\left(P_{i}^{\prime}, P_{-i}\right)$ are two top-2 profiles, then $P_{i}^{\prime}$ is a $\left(P_{i}(1), P_{i}(2)\right)$-swap of $P_{i}$. Let $\mathcal{D}^{n}(2)$ be the set of all top-2 profiles in $\mathcal{D}$. 


\begin{tabular}{ccc|ccc}
$P_{1}$ & $P_{2}$ & $P_{3}$ & $P_{1}^{\prime}$ & $P_{2}$ & $P_{3}$ \\
\hline$a$ & $a$ & $b$ & $b$ & $a$ & $b$ \\
$b$ & $b$ & $a$ & $a$ & $b$ & $a$ \\
$c$ & $c$ & $c$ & $c$ & $c$ & $c$ \\
$d$ & $d$ & $d$ & $d$ & $d$ & $d$
\end{tabular}

Table 3: Weak elementary monotonicity

Definition 14 An scf $f: \mathcal{D}^{n} \rightarrow$ A satisfies weak elementary monotonicity if $f$ restricted to $\mathcal{D}^{n}(2)$ satisfies elementary monotonicity.

Note that weak elementary monotonicity is also a weakening of positive responsiveness property since in the subdomain $\mathcal{D}^{n}(2)$, elementary monotonicity and positive responsiveness is the same. Weak elementary monotonicity applies to very specific preference profiles. First, it requires that $P_{i}^{\prime}$ is a $\left(P_{i}(1), P_{i}(2)\right)$-swap of $P_{i}$. Second, it requires that every agent in $N \backslash\{i\}$ must have either $P_{i}$ or $P_{i}^{\prime}$ as his preference. A typical pair of preference profiles where weak elementary monotonicity can be applied is shown in Table 3 . We assume $A=\{a, b, c, d\}$ and $a \succ b \succ c \succ d$ and $n=3$. Profiles $\left(P_{1}, P_{2}, P_{3}\right)$ and $\left(P_{1}^{\prime}, P_{2}, P_{3}\right)$ in Table 3 differ in agent 1's preference and $P_{1}^{\prime}$ is a $(a, b)$-swap of $P_{i}$. Also, notice that $a$ and $b$ are neighbors in $\succ$, and $P_{2}=P_{1}, P_{3}=P_{1}^{\prime}$ (hence, $P_{2} \neq P_{3}$ ). Weak elementary monotonicity applies to such profiles and requires that if $f\left(P_{1}, P_{2}, P_{3}\right)=b$, then $f\left(P_{1}^{\prime}, P_{2}, P_{3}\right)=b$.

We now state our result for the single peaked domain.

TheOrem 3 Suppose $f: \mathcal{S}^{n} \rightarrow A$ is a unanimous scf, where $\mathcal{S}$ is the single peaked domain. Then, the following statements are equivalent.

1. $f$ is $G$-LOBIC and satisfies weak elementary monotonicity.

2. $f$ is DSIC.

Since weak elementary monotonicity is a relatively weak condition, this result shows how little is required on top of unanimity to get the counterpart of Theorem 1 in the single peaked domain. In other words, though Theorem 1 breaks down in the single peaked domain, the nature of the break down is very nuanced.

The result in Theorem 3 can also be extended to some extensions of single peaked domain. For instance, Demange (1982) defines a notion of single peakedness on a tree graph, which requires single peakedness along paths of a tree graph whose vertices are alternatives. Our result can be easily extended to such a domain. Similarly, it can also be extended to multiple single peaked domain discussed in Reffgen (2015). 


\subsection{Equivalence in a Large Class of Local Domains}

We now provide a generalization of Theorem 3 to a larger class of local domains with the help of an additional axiom.

Definition 15 An scf $f$ is tops-only if for every pair of profiles $\mathbf{P}$ and $\mathbf{P}^{\prime}$ in its domain with $P_{i}(1)=P_{i}^{\prime}(1)$ for all $i \in N$, we have $f(\mathbf{P})=f\left(\mathbf{P}^{\prime}\right)$.

Tops-only property requires that the scf is only sensitive to the top ranked alternatives of each agent. This is a well-studied axiom is social choice theory. In many domains, DSIC and unanimity implies the tops-only property (Weymark, 2008; Saporiti, 2009; Chatterji and Sen, 2011). One of the motivations for using voting mechanisms which satisfy this axiom is computational. Imagine a setting where agents are voting to choose a partition of a set of objects - for instance, geographical districts are partitioned into electoral districts, a society is partitioned into groups of individuals (Fishburn and Rubinstein, 1986; Chambers and Miller, 2011). The number of possible partitions of a finite set of objects is exponential in the number of objects. In such a case, an scf satisfying the tops-only axiom is an attractive option. Topsonlyness and unanimity allows us to weaken positive responsiveness in Theorem 2.

TheOREm 4 Suppose $f: \mathcal{C}^{n} \rightarrow A$ is a tops-only and unanimous scf, where $\mathcal{C}$ is any connected domain without restoration. Then, the following statements are equivalent.

1. $f$ is G-LOBIC and satisfies weak elementary monotonicity.

2. $f$ is DSIC.

The proof of Theorem 4 is given in the Appendix. The tops-only property is required in the above theorem as the following example illustrates.

\section{EXAMPLE 1}

Suppose $A=\{a, b, c, d\}$ and $N=\{1,2,3\}$. Consider the domain of preferences shown in Table 4. Note that it is a connected domain without restoration - in fact, it is a successive single crossing domain (Carroll, 2012), which we formally define in Section 5.5.

We now describe an scf for the domain in Table 4. The scf chooses an alternative if it is the top ranked alternative of all the three agents. In all other profiles, either $a$ or $d$ is chosen depending on the following two cases.

- If $a$ is preferred to $d$ by all the agents, then $a$ is chosen. If $d$ is preferred to $a$ by all the agents, then $d$ is chosen. 


\begin{tabular}{ccccccc}
$P^{1}$ & $P^{2}$ & $P^{3}$ & $P^{4}$ & $P^{5}$ & $P^{6}$ & $P^{7}$ \\
\hline$a$ & $b$ & $b$ & $b$ & $b$ & $d$ & $d$ \\
$b$ & $a$ & $c$ & $c$ & $d$ & $b$ & $c$ \\
$c$ & $c$ & $a$ & $d$ & $c$ & $c$ & $b$ \\
$d$ & $d$ & $d$ & $a$ & $a$ & $a$ & $a$
\end{tabular}

Table 4: A domain where Theorem 4 fails without tops-onlyness.

- If exactly two agents prefer $a$ to $d$ then $d$ is chosen. If exactly two agents prefer $d$ to $a$ then $a$ is chosen.

We denote this scf by $\bar{f}$.

LEMMA 1 The scf $\bar{f}$ defined on the domain in Table 4 is G-LOBIC, unanimous, and satisfies weak elementary monotonicity. However, it is not DSIC and fails tops-onlyness.

The proof is in the Appendix. The intuition behind Lemma 1 is more complicated - the structure of the domain plays an important role.

\subsection{Equivalence in Unrestricted Domain}

We now come to the final result of the paper, which is a strengthening of Theorem 1.

TheOrem 5 Suppose $|A| \geq 3$ and $f: \mathcal{P}^{n} \rightarrow A$ is a $G$-LOBIC scf, where $\mathcal{P}$ is the unrestricted domain. If $f$ satisfies unanimity, then it satisfies elementary monotonicity. Hence, if $f$ is G-LOBIC and unanimous, then it is DSIC.

Theorem 5 is a strengthening of Theorem 1 since we use G-LOBIC instead of G-OBIC. However, one can still deduce the result of Theorem 5 from existing results in the literature as follows. Carroll (2012) notes that his results also hold if we consider Bayesian incentive compatibility. Since he shows local incentive compatibility implies full incentive compatibility in the unrestricted domain, we can conclude that if $f$ is LOBIC (with respect to some prior) then it is OBIC. We can then use Theorem 1 to conclude that if $f$ is G-LOBIC and unanimous, then it must be a dictatorship. Since the proof of Theorem 1 in Majumdar and Sen (2004) directly establishes dictatorship (using induction on the number of agents), our proof provides an alternate and stronger version of their result.

\subsection{Connected Domains without Restoration}

In this section, we identify some domains that are connected without restoration to show that the results in Theorems 2 and 4 apply to a large class of domains. As we noted earlier, 
Sato (2013) has already shown that the unrestricted domain and the single peaked domain are connected without restoration. We present two more domains that are connected without restoration.

Definition 16 A preference ordering $P$ is single dipped with respect to an ordering $\succ$ over alternatives if for every $a, b \in A$ with $a \succ b \succ P(|A|)$ or $P(|A|) \succ b \succ a$, we have $a P b$.

The single dipped domain is important in studying various practical problems including the problem of locating a public "bad". It admits various interesting DSIC mechanisms (Klaus et al., 1997; Peremans and Storcken, 1999; Barberà et al., 2012; Manjunath, 2014). Notice that there are only two alternatives (the maximal and the minimal alternatives in $\succ$ ) which can be top ranked in any single dipped preference. The set of all single dipped preferences is denoted by $\overline{\mathcal{S}}$.

LEMMA 2 The domain $\overline{\mathcal{S}}$ is a connected domain without restoration.

Proof: Take any preference ordering $P$, and denote by $\bar{P}$ the reverse of the preference ordering $P$, i.e., $\bar{P}(j)=P(|A|-j+1)$ for all $j$. Note that $P$ is single dipped with respect to $\succ$ if and only if $\bar{P}$ is single peaked with respect to $\succ$. Now, consider two single dipped preference orderings $P$ and $P^{\prime}$. Since the single peaked domain is connected without restoration, there is a sequence of distinct single peaked preferences without restoration between $\bar{P}$ and $\bar{P}^{\prime}$. By taking the reverse of each preference ordering in this sequence, we get the desired sequence without restoration between $P$ and $P^{\prime}$.

Another important class of domains that can be shown to be connected without restoration is a successive single crossing domains in Carroll (2012).

Definition 17 A set of preferences $\mathcal{D}$ is a single crossing domain if there exists a strict linear order $\succ$ on the set of alternatives and a strict linear order $\triangleleft$ on the set of preferences $\mathcal{D}$ such that for all $a, b \in A$ and for all $P, P^{\prime} \in \mathcal{D}$,

- $a \succ b, P \triangleleft P^{\prime}$, and $a P b$ implies $a P^{\prime} b$

- $a \succ b, P \triangleleft P^{\prime}$, and $b P^{\prime} a$ implies $b P a$.

Single crossing domains are a well studied domain in voting and political economy since they ensure existence of a Condorcet winner (Saporiti, 2009).

For any ordering $P$ over $A$ and any ordering $\succ$ over $A$, let $X(P, \succ):=\{(a, b): a \succ b, a P b\}$. Clearly, a set of preferences $\mathcal{D}$ is a single crossing domain if and only if there exists a strict 


\begin{tabular}{c|c|c|c}
$P^{1}$ & $P^{2}$ & $P^{3}$ & $P^{4}$ \\
\hline$a$ & $a$ & $c$ & $c$ \\
$b$ & $c$ & $a$ & $b$ \\
$c$ & $b$ & $b$ & $a$
\end{tabular}

Table 5: A successive single crossing domain

linear order $\succ$ on the set of alternatives and a strict linear order $\triangleleft$ on the set of preferences $\mathcal{D}$ such that for any $P, P^{\prime} \in \mathcal{D}$ with $P \triangleleft P^{\prime}$, we have $X(P, \succ) \subsetneq X\left(P^{\prime}, \succ\right)$ (notice the strict inclusion). We will denote a single crossing domain as $\mathcal{D}^{\succ} \triangleleft$.

A single crossing domain $\mathcal{D}^{\succ, \triangleleft}:=\left\{P^{1}, \ldots, P^{l}\right\}$ with $P^{1} \triangleleft \ldots \triangleleft P^{l}$ is a successive single crossing domain if for every $j \in\{1, \ldots, l-1\},\left|X\left(P^{j}, \succ\right)\right|+1=\left|X\left(P^{j+1}, \succ\right)\right|$. Successive single crossing domains were introduced in Carroll (2012).

Let $A=\{a, b, c\}$. Table 5 gives an example of a successive single crossing domain. This domain is single crossing with respect to $c \succ b \succ a$ and $P^{1} \triangleleft P^{2} \triangleleft P^{3} \triangleleft P^{4}$. By comparing with the single peaked domain in Table 2, one sees that these two domains can be quite different - see more discussions on this in Saporiti (2009).

LEMMA 3 A successive single crossing domain is connected without restoration.

Proof: $\quad$ Let $\mathcal{D}^{\succ} \triangleleft:=\left\{P^{1}, \ldots, P^{l}\right\}$ be a successive single crossing domain with $P^{1} \triangleleft P^{2} \triangleleft$ $\ldots \triangleleft P^{l}$. Pick $P^{j}, P^{k} \in \mathcal{D}^{\succ} \triangleleft$ with $j<k$. The sequence of preferences $\left(P^{j}, P^{j+1}, \ldots, P^{k}\right)$ satisfies the fact that for any $j^{\prime} \in\{j, j+1, \ldots, k-1\}, P^{j^{\prime}}$ and $P^{j^{\prime}+1}$ are adjacent - this follows from the definition of a successive single crossing domain. Now, assume for contradiction, there is some pair of alternatives $x, y \in A$ such that they are swapped more than once in this sequence. But the single crossing property requires that if $x \succ y$, once $x P^{k^{\prime}} y$ for some $P^{k^{\prime}}$ in the sequence, it must remain $x P^{l^{\prime}} y$ for all $l^{\prime}>k^{\prime}$. Hence, getting swapped more than once will violate the single crossing property. This means that every successive single crossing domain is connected without restoration.

Note that not every connected domain without restoration satisfies richness. The single dipped domain is not a rich domain. Further, the successive single crossing domain in Table 5 is not rich. However, there are rich successive single crossing domains (Mishra et al., 2015). 


\section{Discussions on Elementary Monotonicity}

The crucial condition in Theorem 2 is elementary monotonicity. The scf $f^{*}$ discussed in Proposition 1 is an example of an scf that satisfies G-LOBIC but fails elementary monotonicity. Hence, we cannot hope to drop elementary monotonicity in Theorem 2.

As discussed in the literature, elementary monotonicity is satisfied by variety of scfs (Moulin, 1983; Majumdar and Sen, 2004). However, it is admittedly a strong enough condition along with G-LOBIC to imply DSIC in local domains. The positive responsiveness property is equivalent to elementary monotonicity in a large class of local domains. Both these conditions are weaker versions of Maskin monotonicity, which is known to be necessary and sufficient for dominant strategy incentive compatibility in the unrestricted domain (Kalai and Muller, 1977). To see why elementary monotonicity or positive responsiveness is much weaker than Maskin monotonicity, note that these axioms are satisfied by a large class of scfs but Maskin monotonicity and unanimity implies dictatorship in the unrestricted domain.

Since all the additional axioms that we have used are weak, they are useful to rule out scfs that are not G-LOBIC in many domains. We give two examples. Consider the status-quo scf, which is specified by a status-quo alternative. It chooses the status-quo alternative at all the preference profiles except when everyone's top ranked alternative is the same. When everyone's top ranked alternative is the same, then that alternative is chosen. This scf clearly satisfies positive responsiveness, elementary monotonicity, and unanimity. However, it is not DSIC in many domains (including the single peaked domain). We can then conclude from our results that the status-quo scf cannot be G-LOBIC in those domains.

Similarly, some scoring rules cannot be G-LOBIC. It is easy to see that all scoring rules satisfy elementary monotonicity. But scoring rules may not be DSIC in many interesting domains. To see this, consider the single peaked domain in Table 2. Consider a scoring rule in this domain where the top ranked alternative gets a score of 2 , the second ranked alternative gets a score of 1 , and the last ranked alternative gets a score of 0 . If there is a tie in the score, we break the tie using a linear order $\triangleright$, where $a \triangleright b \triangleright c$. Now, suppose agent 1 has preference where $a$ is top ranked, $b$ is second ranked, and $c$ is third ranked. Further, agents 2 and 3 have identical preference, where $c$ is top, $b$ is second, and $a$ is third. The outcome of the scoring rule at this profile is $c$. But agent 1 can get the outcome $b$ if he reports a preference ordering where $b$ is top. Hence, this scoring rule is not DSIC. Using our results, we can conclude that such a scoring rule is not G-LOBIC in the single peaked domain. 
We also point out that elementary monotonicity plays an important role in the analysis of OBIC scfs with uniform priors and correlated priors. Majumdar and Sen (2004) show that when agents have uniform priors, every neutral scf satisfying elementary monotonicity is OBIC in the unrestricted domain. Thus, they show that a large class of scfs that are not DSIC are OBIC with uniform priors in the unrestricted domain. A similar result is shown with correlated priors in Bhargava et al. (2014). In contrast, Theorem 2 shows a negative implication of elementary monotonicity under independent generic priors.

\section{APPENDIX}

\section{Proof of Proposition 1}

Clearly, $f^{*}$ is unanimous and anonymous. However, $f^{*}$ is not DSIC. To see this, consider a profile $\mathbf{P}$ such that for all agents $i \neq 3$, we have $P_{i}(1)=a$ - note that since $a$ is the leftmost alternative, there is a unique preference ordering where $a$ is top ranked. For agent 3, pick any preference ordering where $b$ is preferred to $a$. As a result, $f^{*}(\mathbf{P})=b$. A possible profile is shown in Table 6 - note that $a P_{1} b$. Now, fixing the preference profile of all agents except agent 1 , if agent 1 reports a preference ordering $P_{1}^{\prime}$ such that $P_{1}^{\prime}(1)=b, P_{1}^{\prime}(2)=a$, then $f^{*}$ will choose $a$ - see Table 6 . Hence, agent 1 can manipulate.

\begin{tabular}{ccc|ccc}
$P_{1}$ & $P_{2}$ & $P_{3}$ & $P_{1}^{\prime}$ & $P_{2}$ & $P_{3}$ \\
\hline$a$ & $a$ & $b$ & $b$ & $a$ & $b$ \\
$b$ & $b$ & $a$ & $a$ & $b$ & $a$ \\
$c$ & $c$ & $c$ & $c$ & $c$ & $c$
\end{tabular}

Table 6: Failure of DSIC of $f^{*}$

However, we show that $f^{*}$ is G-OBIC. Let the prior of each agent $i$ be given by the map $\mu_{i}: \mathcal{S} \rightarrow(0,1)$. To show that $f^{*}$ is OBIC with respect to $\left\{\mu_{i}\right\}_{i \in N}$ such that these are generic priors, we will compute the interim outcome probabilities of every agent in $\{1,2,3\}$.

If an agent $i \in\{1,2,3\}$ has preference $P_{i}$ then define

$$
O_{i}^{f^{*}}\left(P_{i}\right):=\left\{x \in A: f^{*}\left(P_{i}, P_{-i}\right)=x \text { for some } P_{-i}\right\}
$$

By definition of $f^{*}$, for every $i \in\{1,2,3\}$ and for every $P_{i} \in \mathcal{S}, O_{i}^{f^{*}}\left(P_{i}\right) \subseteq\left\{a, b, P_{i}(1)\right\}$, and if $P_{i}(1) \neq c$, then $O_{i}^{f^{*}}\left(P_{i}\right)=\{a, b\}$. Let $\bar{P}$ be the unique preference ordering where the leftmost alternative $a$ is top ranked. Denote the probability that agent $i$ has preference $\bar{P}$ as $q_{i} \equiv \mu_{i}(\bar{P})$. Similarly, denote by $\hat{q}_{i} \equiv \mu_{i}(\hat{P})$, where $\hat{P}$ is the unique ordering where 
alternative $c$ is top ranked. We fix an agent $i \in\{1,2,3\}$ and denote the other two agents in $\{1,2,3\}$ as $j$ and $k$. We consider three possible cases.

Case 1. Suppose $P_{i}=\bar{P}$. Note that $O_{i}^{f^{*}}\left(P_{i}\right)=\{a, b\}$. Then his interim outcome probability for alternative $a$ can be computed as follows. Note that $f^{*}\left(P_{i}, P_{j}, P_{k}\right) \neq a$ if $P_{j}$ and $P_{k}$ are such that either $\left(a P_{j} b\right.$ and $\left.b P_{k} a\right)$ or $\left(a P_{k} b\right.$ and $\left.b P_{j} a\right)$. The probability of this event is

$$
q_{j}\left(1-q_{k}\right)+q_{k}\left(1-q_{j}\right)
$$

Here, we used the fact that the probability that agent $j$ has preference $P_{j}$ such that $b P_{j} a$ is just $\left(1-q_{j}\right)$ and, similarly, the probability that agent $k$ has preference $P_{k}$ such that $b P_{k} a$ is $\left(1-q_{k}\right)$. Since $f^{*}\left(P_{i}, P_{j}, P_{k}\right) \in\{a, b\}$, the interim outcome probability of choosing $a$ at $P_{i}$ for agent $i$ is

$$
1-q_{j}-q_{k}+2 q_{j} q_{k}
$$

and the interim outcome probability of choosing $b$ at $P_{i}$ for agent $i$ is

$$
q_{j}+q_{k}-2 q_{j} q_{k}
$$

Case 2. Suppose $P_{i}$ is such that $P_{i}(1)=b$ - this is possible for two preference orderings. Note that $O_{i}^{f^{*}}\left(P_{i}\right)=\{a, b\}$. Then, his interim outcome probability for alternative $b$ can be computed as follows. Note that $f^{*}\left(P_{i}, P_{j}, P_{k}\right) \neq b$ if $P_{j}$ and $P_{k}$ are such that either $\left(a P_{j} b\right.$ and $\left.b P_{k} a\right)$ or $\left(a P_{k} b\right.$ and $\left.b P_{j} a\right)$. The probability of this event is

$$
q_{j}\left(1-q_{k}\right)+q_{k}\left(1-q_{j}\right)
$$

Hence, the interim outcome probability of choosing $b$ at $P_{i}$ for agent $i$ is

$$
1-q_{j}-q_{k}+2 q_{j} q_{k}
$$

Since $O_{i}^{f^{*}}\left(P_{i}\right)=\{a, b\}$, the interim outcome probability of choosing $a$ at $P_{i}$ for agent $i$ is

$$
q_{j}+q_{k}-2 q_{j} q_{k}
$$

CASE 3. Suppose $P_{i}$ is such that $P_{i}(1)=c$, i.e., $P_{i}=\hat{P}$. Then, $O_{i}^{f^{*}}\left(P_{i}\right)=\{a, b, c\}$. His interim outcome probability for alternative $c$ can be computed straightforwardly $-c$ is chosen if and only if $P_{j}(1)=P_{k}(1)=P_{i}(1)=c$. This is possible if and only if both agents $j$ and $k$ have the preference $\hat{P}$. The probability of this event is $\hat{q}_{j} \hat{q}_{k}$. Hence, the interim outcome probability for alternative $P_{i}(1)$ at $P_{i}$ for agent $i$ is $\hat{q}_{j} \hat{q}_{k}$. Next, the interim outcome 
probability for agent $i$ for alternative $a$ can be computed as follows. For this, note that $c P_{i} b P_{i} a$. Hence, $f^{*}\left(P_{i}, P_{j}, P_{k}\right)=a$ if $P_{j}$ and $P_{k}$ are such that either $\left(a P_{j} b\right.$ and $\left.b P_{k} a\right)$ or $\left(a P_{k} b\right.$ and $\left.b P_{j} a\right)$ - notice that these events ensure that tops of all agents are not the same and $a$ is not dominated by $b$. The probability of this event is

$$
q_{j}\left(1-q_{k}\right)+q_{k}\left(1-q_{j}\right) .
$$

Hence, the interim outcome probability of choosing $a$ at $P_{i}$ for agent $i$ is

$$
q_{j}+q_{k}-2 q_{j} q_{k}
$$

Since $O_{i}^{f^{*}}\left(P_{i}\right)=\left\{a, b, P_{i}(1)\right\}$, the interim outcome probability of choosing $b$ at $P_{i}$ for agent $i$ is

$$
1-q_{j}-q_{k}+2 q_{j} q_{k}-\hat{q}_{j} \hat{q}_{k}
$$

We enumerate all the interim outcome probabilities in Table 7 by considering the three cases for an agent $i \in\{1,2,3\}$ by denoting the other two agents as $j$ and $k$.

\begin{tabular}{|l|l|l|l|}
\hline & Case 1: $P_{i}=\bar{P}$ & Case $2: P_{i}(1)=b$ & Case $3: P_{i}=\hat{P}$ \\
\hline$\pi_{i}^{f^{*}}\left(a, P_{i}\right)$ & $1-q_{j}-q_{k}+2 q_{j} q_{k}$ & $q_{j}+q_{k}-2 q_{j} q_{k}$ & $q_{j}+q_{k}-2 q_{j} q_{k}$ \\
\hline$\pi_{i}^{f^{*}}\left(b, P_{i}\right)$ & $q_{j}+q_{k}-2 q_{j} q_{k}$ & $1-q_{j}-q_{k}+2 q_{j} q_{k}$ & $1-q_{j}-q_{k}+2 q_{j} q_{k}-\hat{q}_{j} \hat{q}_{k}$ \\
\hline$\pi_{i}^{f^{*}}\left(c, P_{i}\right)$ & 0 & 0 & $\hat{q}_{j} \hat{q}_{k}$ \\
\hline
\end{tabular}

Table 7: Interim outcome probabilities

Now, notice from Table 7 that if agent $i$ has preference $P_{i} \equiv \bar{P}$, the only OBIC constraint to satisfy is

$$
1-q_{j}-q_{k}+2 q_{j} q_{k} \geq q_{j}+q_{k}-2 q_{j} q_{k} .
$$

Alternatively, we must have

$$
\left(1-2 q_{j}\right)\left(1-2 q_{k}\right) \geq 0 .
$$

This prevents any manipulation of agent $i$ to a preference in Case 2 or Case 3. Also, Inequality 1 ensures OBIC constraints when agent $i$ has a preference $P_{i}$ such that $P_{i}(1)=b$. Finally, Inequality 1 also ensures OBIC constraints when agent $i$ has a preference $P_{i}$ such that $P_{i}(1)=c$ - this can be verified by checking from Table 7 that the truthtelling lottery first-order stochastic dominates other lotteries as long as Inequality 1 is satisfied.

Hence, $f^{*}$ is $\mathrm{OBIC}$ if and only if the priors of agents 1,2 , and 3 satisfy

$$
\begin{aligned}
& \left(1-2 q_{2}\right)\left(1-2 q_{3}\right) \geq 0, \\
& \left(1-2 q_{1}\right)\left(1-2 q_{3}\right) \geq 0, \\
& \left(1-2 q_{1}\right)\left(1-2 q_{2}\right) \geq 0 .
\end{aligned}
$$


This is satisfied if and only if $q_{1}, q_{2}, q_{3} \in(0,0.5)$ or $q_{1}, q_{2}, q_{3} \in(0.5,1)$. It also puts no restriction on the probabilities of orderings in $\mathcal{S} \backslash\{\bar{P}\}$. Hence, the set of priors of all agents satisfying $q_{1}, q_{2}, q_{3} \in(0,0.5)$ or $q_{1}, q_{2}, q_{3} \in(0.5,1)$ is a full dimensional subset of the set of all independent priors. As a result, it must have a non-empty intersection with the set of independent generic priors.

\section{Proof of Theorem 2}

The proof is done by establishing two important lemmas. We start by identifying a property that is implied by G-LOBIC.

Definition 18 An scf $f$ satisfies swap monotonicity if for every $i \in N$, for every $P_{i}, P_{i}^{\prime} \in$ $\mathcal{D}$, where $P_{i}^{\prime}$ is an $(a, b)$-swap of $P_{i}$, we have for every $P_{-i} \in \mathcal{D}^{n-1}$,

- $f\left(P_{i}^{\prime}, P_{-i}\right)=f\left(P_{i}, P_{-i}\right)$ if $f\left(P_{i}, P_{-i}\right) \notin\{a, b\}$,

- $f\left(P_{i}^{\prime}, P_{-i}\right) \in\{a, b\}$ if $f\left(P_{i}, P_{-i}\right) \in\{a, b\}$.

Our first claim shows the necessity of swap monotonicity.

LEMMA 4 If an scf is G-LOBIC, then it satisfies swap monotonicity.

Proof: Let $f$ be an LOBIC scf with respect to independent generic priors $\left\{\mu_{i}\right\}_{i \in N}$. For this, consider agent $i \in N$, and pick two preference orderings $P_{i}$ and $P_{i}^{\prime}$ such that $P_{i}^{\prime}$ is an $(a, b)$ swap of $P_{i}$. By definition $P_{i}(k)=a, P_{i}(k+1)=b$ and $P_{i}^{\prime}(k+1)=a, P_{i}^{\prime}(k)=b$ for some $k$ and $P_{i}(j)=P_{i}^{\prime}(j)$ for all $j \notin\{k, k+1\}$. We will do the proof in three steps.

SteP 1. Consider an alternative $x \in A \backslash\{a, b\}$ such that $P_{i}\left(k^{\prime}\right)=P_{i}^{\prime}\left(k^{\prime}\right)=x$, where $k^{\prime}<k$. We will show that $\left\{P_{-i} \in \mathcal{D}^{n-1}: f\left(P_{i}, P_{-i}\right)=x\right\}=\left\{P_{-i} \in \mathcal{D}^{n-1}: f\left(P_{i}^{\prime}, P_{-i}\right)=x\right\}$. We do this using induction on $k^{\prime}$. If $k^{\prime}=1$, by observing that $P_{i}\left(k^{\prime \prime}\right)=P_{i}^{\prime}\left(k^{\prime \prime}\right)$ for all $k^{\prime \prime}<k$, LOBIC implies that

$$
\begin{aligned}
\sum_{P_{-i}: f\left(P_{i}, P_{-i}\right)=P_{i}(1)} \mu\left(P_{-i}\right) & \geq \sum_{P_{-i}: f\left(P_{i}^{\prime}, P_{-i}\right)=P_{i}(1)} \mu\left(P_{-i}\right) \\
\sum_{P_{-i}: f\left(P_{i}^{\prime}, P_{-i}\right)=P_{i}^{\prime}(1)} \mu\left(P_{-i}\right) & \geq \sum_{P_{-i}: f\left(P_{i}, P_{-i}\right)=P_{i}^{\prime}(1)} \mu\left(P_{-i}\right) .
\end{aligned}
$$

Combining these inequalities, we get

$$
\sum_{P_{-i}: f\left(P_{i}, P_{-i}\right)=P_{i}(1)} \mu\left(P_{-i}\right)=\sum_{P_{-i}: f\left(P_{i}^{\prime}, P_{-i}\right)=P_{i}(1)} \mu\left(P_{-i}\right) .
$$


Since priors are generic, we get that

$$
\left\{P_{-i}: f\left(P_{i}, P_{-i}\right)=P_{i}(1)\right\}=\left\{P_{-i}: f\left(P_{i}^{\prime}, P_{-i}\right)=P_{i}(1)\right\} .
$$

Now, suppose the claim is true for all $k^{\prime \prime}<k^{\prime}$. Notice that the top $k^{\prime}$ alternatives in $P_{i}$ and $P_{i}^{\prime}$ are the same - denote this set as $B$. Now, we apply LOBIC to top $k^{\prime}$ alternatives in $P_{i}$ and $P_{i}^{\prime}$ to get

$$
\begin{aligned}
\sum_{P_{-i}: f\left(P_{i}, P_{-i}\right) \in B} \mu_{i}\left(P_{-i}\right) & \geq \sum_{P_{-i}: f\left(P_{i}^{\prime}, P_{-i}\right) \in B} \mu_{i}\left(P_{-i}\right) \\
\sum_{P_{-i}: f\left(P_{i}^{\prime}, P_{-i}\right) \in B} \mu_{i}\left(P_{-i}\right) & \geq \sum_{P_{-i}: f\left(P_{i}, P_{-i}\right) \in B} \mu_{i}\left(P_{-i}\right) .
\end{aligned}
$$

Using genericity of $\mu_{i}$ gives us

$$
\left\{P_{-i}: f\left(P_{i}, P_{-i}\right) \in B\right\}=\left\{P_{-i}: f\left(P_{i}^{\prime}, P_{-i}\right) \in B\right\} .
$$

Using the induction hypothesis, we have for all $k^{\prime \prime}<k^{\prime}$,

$$
\left\{P_{-i}: f\left(P_{i}, P_{-i}\right)=P_{i}\left(k^{\prime \prime}\right)\right\}=\left\{P_{-i}: f\left(P_{i}^{\prime}, P_{-i}\right)=P_{i}\left(k^{\prime \prime}\right)\right\} .
$$

Hence, we get

$$
\left\{P_{-i}: f\left(P_{i}, P_{-i}\right)=P_{i}\left(k^{\prime}\right)\right\}=\left\{P_{-i}: f\left(P_{i}^{\prime}, P_{-i}\right)=P_{i}\left(k^{\prime}\right)\right\} .
$$

SteP 2. In this step, we show that $\left\{P_{-i}: f\left(P_{i}, P_{-i}\right) \in\{a, b\}\right\}=\left\{P_{-i}: f\left(P_{i}^{\prime}, P_{-i}\right) \in\{a, b\}\right\}$. Applying LOBIC, we get

$$
\begin{aligned}
\sum_{P_{-i}: f\left(P_{i}, P_{-i}\right) \in B\left(b, P_{i}\right)} \mu_{i}\left(P_{-i}\right) & \geq \sum_{P_{-i}: f\left(P_{P}^{\prime}, P_{-i}\right) \in B\left(b, P_{i}\right)} \mu_{i}\left(P_{-i}\right) \\
\sum_{P_{-i}: f\left(P_{i}^{\prime}, P_{-i}\right) \in B\left(a, P_{i}^{\prime}\right)} \mu_{i}\left(P_{-i}\right) & \geq \sum_{P_{-i}: f\left(P_{i}, P_{-i}\right) \in B\left(a, P_{i}^{\prime}\right)} \mu_{i}\left(P_{-i}\right) .
\end{aligned}
$$

Since $B\left(b, P_{i}\right)=B\left(a, P_{i}^{\prime}\right)$, by genericity we get

$$
\left\{P_{-i}: f\left(P_{i}, P_{-i}\right) \in B\left(b, P_{i}\right)\right\}=\left\{P_{-i}: f\left(P_{i}^{\prime}, P_{-i}\right) \in B\left(b, P_{i}\right)\right\} .
$$

By Step 1, this implies that $\left\{P_{-i}: f\left(P_{i}, P_{-i}\right) \in\{a, b\}\right\}=\left\{P_{-i}: f\left(P_{i}^{\prime}, P_{-i}\right) \in\{a, b\}\right\}$.

SteP 3. Consider an alternative $x \in A \backslash\{a, b\}$ such that $P_{i}\left(k^{\prime}\right)=P_{i}^{\prime}\left(k^{\prime}\right)=x$, where $k^{\prime}>k+1$. Using the facts in Steps 1 and 2, we can mimic the method in Step 1 to show that $\left\{P_{-i} \in \mathcal{D}^{n-1}: f\left(P_{i}, P_{-i}\right)=x\right\}=\left\{P_{-i} \in \mathcal{D}^{n-1}: f\left(P_{i}^{\prime}, P_{-i}\right)=x\right\}$.

Steps 1,2 , and 3 show that $f$ satisfies swap monotonicity.

Note that Lemma 4 holds in any arbitrary domain. We now use this to prove the following result. 
Lemma 5 Suppose $\mathcal{D}$ is any domain and $f: \mathcal{D}^{n} \rightarrow A$ is an scf on this domain. Then, the following statements are equivalent.

1. $f$ is $G$-LOBIC and satisfies elementary monotonicity.

2. $f$ is $L D S I C$.

Proof: First, we show that a LDSIC scf $f$ satisfies elementary monotonicity. To see this, consider $i \in N$ and $P_{-i}$. Let $P_{i}$ and $P_{i}^{\prime}$ be two preferences in $\mathcal{D}$ such that $P_{i}^{\prime}$ is an $(a, b)$-swap of $P_{i}$ and $f\left(P_{i}, P_{-i}\right)=b$. Assume for contradiction that $f\left(P_{i}^{\prime}, P_{-i}\right)=c \neq b$. If $c P_{i} b$, then $i$ can manipulate from $P_{i}$ to $P_{i}^{\prime}$. If $b P_{i} c$, then, by construction, $b P_{i}^{\prime} c$, and again, agent $i$ can manipulate from $P_{i}^{\prime}$ to $P_{i}$. This is a contradiction.

Further, an LDSIC scf is LOBIC with respect to all priors, and hence, it is G-LOBIC. Now, we show that if $f: \mathcal{D}^{n} \rightarrow A$ is G-LOBIC and satisfies elementary monotonicity, then it is LDSIC. Fix an agent $i$ and $P_{-i}$. Now, pick two preference orderings $P_{i}$ and $P_{i}^{\prime}$ such that $P_{i}^{\prime}$ is an $(a, b)$-swap of $P_{i}$, where $P_{i}(k)=a, P_{i}(k+1)=b$ and $P_{i}^{\prime}(k+1)=a, P_{i}^{\prime}(k)=b$ for some $k$. Suppose $f\left(P_{i}, P_{-i}\right)=x$ and $f\left(P_{i}^{\prime}, P_{-i}\right)=y$. Suppose $P_{i}\left(k^{\prime}\right)=x$. If $k^{\prime}<k$ or $k^{\prime}>k+1$, then by Lemma 4 , we have $y=x$ (swap monotonicity). So, agent $i$ cannot manipulate from $P_{i}$ to $P_{i}^{\prime}$. If $k^{\prime}=k$, then $x=a$, and by swap monotonicity $f\left(P_{i}^{\prime}, P_{-i}\right) \in\{a, b\}$. Since $a P_{i} b$, agent $i$ cannot manipulate from $P_{i}$ to $P_{i}^{\prime}$. The other possibility is $k^{\prime}=k+1$. In that case, $x=b$, and elementary monotonicity ensures that $f\left(P_{i}^{\prime}, P_{-i}\right)=b$. Hence, agent $i$ cannot manipulate from $P_{i}$ to $P_{i}^{\prime}$. This shows that $f$ is LDSIC.

Proof of Theorem 2:

Equivalence of (1) AND (2). This equivalence follows from Lemma 5 because in local domains an LDSIC scf is DSIC.

EQUivalenCE OF (2) AND (3). If $\mathcal{D}$ is a rich connected domain without restoration, we first show that a G-LOBIC $f$ satisfying elementary monotonicity also satisfies positive responsiveness. To see this, fix agent $i \in N, P_{-i} \in \mathcal{D}^{n-1}$ and $P_{i}, P_{i}^{\prime} \in \mathcal{D}$ such that $f\left(P_{i}, P_{-i}\right)=a$ and $P_{i}^{\prime}(1)=a$. Since $\mathcal{D}$ is connected without restoration there is a connected sequence $\left(P^{0} \equiv P_{i}, P^{1}, \ldots, P^{k} \equiv P_{i}^{\prime}\right)$. Consider $P^{j}$ and $P^{j+1}$ in this sequence. By construction, $P^{j+1}$ is a $\left(x^{j}, y^{j}\right)$-swap of $P^{j}$ for some pair of alternatives $x^{j}, y^{j}$. If $f\left(P^{j}, P_{-i}\right) \notin\left\{x^{j}, y^{j}\right\}$, then $f\left(P^{j}, P_{-i}\right)=f\left(P^{j+1}, P_{-i}\right)$ by Lemma 4 . By elementary monotonicity, if $f\left(P^{j}, P_{-i}\right)=y^{j}$, then $f\left(P^{j+1}, P_{-i}\right)=y^{j}=f\left(P^{j}, P_{-i}\right)$. We argue that $f\left(P^{j}, P_{-i}\right) \neq x^{j}$, and we will be done. 
To see this, note that since $\mathcal{D}$ is a connected domain without restoration, no swap along the sequence $\left(P^{0} \equiv P_{i}, P^{1}, \ldots, P^{k} \equiv P_{i}^{\prime}\right)$ is repeated. Since $P_{i}^{\prime}(1)=a$, connectedness without restoration implies that the rank of $a$ along this sequence can never go down. Hence, $a \neq x^{j}$ for any $j$. Hence, $f\left(P^{0}, P_{-i}\right) \neq x^{0}$. This implies that $f\left(P^{1}, P_{-i}\right)=a$. Using induction, if we assume $f\left(P^{j}, P_{-i}\right)=a$ and since $a \neq x^{j}$, we get $f\left(P^{j+1}, P_{-i}\right)=a$, and we are done.

For the converse, suppose $f$ is G-LOBIC and satisfies positive responsiveness. We will show that $f$ satisfies elementary monotonicity. Fix agent $i \in N, P_{-i} \in \mathcal{D}^{n-1}$ and $P_{i}, P_{i}^{\prime} \in \mathcal{D}$ such that $P_{i}^{\prime}$ is an $(a, b)$-swap of $P_{i}$ with $f\left(P_{i}, P_{-i}\right)=b$. Note that $b P_{i}^{\prime} a$. Assume for contradiction that $f\left(P_{i}^{\prime}, P_{-i}\right) \neq b$. By Lemma $4, f\left(P_{i}^{\prime}, P_{-i}\right)=a$. Since $\mathcal{D}$ is rich, there is a preference ordering $P_{i}^{\prime \prime}$ with $P_{i}^{\prime \prime}(1)=b$. Further, since the domain is connected without restoration, there is a connected sequence $\left(P^{0} \equiv P_{i}^{\prime}, P^{1}, \ldots, P^{K} \equiv P_{i}^{\prime \prime}\right)$.

For any $P^{j}$ in this sequence, define as before $B\left(b, P^{j}\right)=\left\{x \in A: x P^{j} b\right.$ or $\left.x=b\right\}$. We show that for any $P^{j}$ in this sequence, $f\left(P^{j}, P_{-i}\right) \notin B\left(b, P^{j}\right)$. Since $f\left(P^{0}, P_{-i}\right)=a$, the claim is true for $j=0$. Suppose the claim is true for all the preferences in the sequence till $P^{k}$. Suppose $P^{k+1}$ is a $(x, y)$-swap of $P^{k}$.

Consider the case when $\{x, y\} \subseteq B\left(b, P^{k}\right)$. Since $f\left(P^{k}, P_{-i}\right) \notin B\left(b, P^{k}\right)$, Lemma 4 implies that $f\left(P^{k}, P_{-i}\right)=f\left(P^{k+1}, P_{-i}\right)$, which further implies that $f\left(P^{k+1}, P_{-i}\right) \notin B\left(b, P^{k+1}\right)$.

Now, consider the case when $\{x, y\} \subseteq\left(A \backslash B\left(b, P^{k}\right)\right)$. By Lemma $4, f\left(P^{k+1}, P_{-i}\right) \notin$ $B\left(b, P^{k+1}\right)$.

The only case that remains is $x=b$ and $y \notin B\left(b, P^{k}\right)$, i.e., $y$ is just below $b$ in $P^{k}$. Since $\mathcal{D}$ is connected without restoration and the connected sequence $\left(P^{0} \equiv P_{i}^{\prime}, P^{1}, \ldots, P^{k} \equiv P_{i}^{\prime \prime}\right)$ satisfies $P_{i}^{\prime \prime}(1)=b$, the rank of $b$ can never go down along this sequence. Hence, $b \neq x$. Hence, this case is not possible.

Hence, by induction $f\left(P_{i}^{\prime \prime}, P_{-i}\right) \notin B\left(b, P_{i}^{\prime \prime}\right)=\{b\}$. But the fact that $f\left(P_{i}, P_{-i}\right)=b$ and $P_{i}^{\prime \prime}(1)=b$ implies that $f$ does not satisfy positive responsiveness, a contradiction.

\section{Proof of Corollary 2}

We say an scf $f$ is Pareto efficient if at every profile of preferences $\mathbf{P}$ with $a P_{i} b$ for all $i \in N$, we have $f(\mathbf{P}) \neq b$. We first show that if the domain is rich connected without restoration, then a unanimous and G-LOBIC scf $f$ is Pareto efficient. Note here that since $f$ is G-LOBIC, 
it satisfies swap monotonicity (Lemma 4). To show this, let $\mathbf{P}$ be a profile with $a P_{i} b$ for all $i \in N$ and $f(\mathbf{P})=b$.

Now, chosse any $i \in N$. By richness, there is a preference ordering $P_{i}^{\prime}$ for every $i$ such that $P_{i}^{\prime}(1)=a$. Moreover, by connectedness without restoration, there is a sequence of preferences $\left(P_{i}=P_{i}^{0}, P_{i}^{1}, \ldots, P_{i}^{K} \equiv P_{i}^{\prime}\right)$. We argue that $f\left(P_{i}^{j}, P_{-i}\right) \notin B\left(a, P_{i}^{j}\right)$ for all $P_{i}^{j}$ in the sequence. Since $f\left(P_{i}^{0}, P_{-i}\right)=b$ and $a P_{i}^{0} b$, the claim is true for $P_{i}^{0}$. Now, assume that the claim is true for some preference $P_{i}^{k}$ in the sequence. Note that by the without restoration property, the rank of $a$ can never go down along this sequence. Hence, if $P_{i}^{k+1}$ is a $(x, a)$-swap of $P_{i}^{k}$, then $x \in B\left(a, P_{i}^{k}\right)$. By our induction hypothesis, $x \neq f\left(P_{i}^{k}, P_{-i}\right)$. By swap monotonicity, $f\left(P_{i}^{k}, P_{-i}\right)=f\left(P_{i}^{k+1}, P_{-i}\right)$. If the swap from $P_{i}^{k}$ to $P_{i}^{k+1}$ involves a pair of alternatives which belong to $B\left(a, P_{i}^{k}\right)$, swap monotonicity again gives $f\left(P_{i}^{k}, P_{-i}\right)=f\left(P_{i}^{k+1}, P_{-i}\right)$. If the swap from $P_{i}^{k}$ to $P_{i}^{k+1}$ involves a pair of alternatives which lie outside $B\left(a, P_{i}^{k}\right)$, swap monotonicity again gives $f\left(P_{i}^{k+1}, P_{-i}\right) \notin B\left(a, P_{i}^{k+1}\right)$. Hence, in all cases, we have $f\left(P_{i}^{k+1}, P_{-i}\right) \notin B\left(a, P_{i}^{k+1}\right)$. This, $f\left(P_{i}^{\prime}, P_{-i}\right) \notin B\left(a, P_{i}^{\prime}\right)=\{a\}$.

We repeat this procedure for all the agents to reach a preference profile $\mathbf{P}^{\prime}$ such that $P_{i}^{\prime}(1)=a$ and $f\left(\mathbf{P}^{\prime}\right)=a$, which contradicts unanimity.

Now, since $n=2$, fix $i \in\{1,2\}$ and suppose $P_{i}^{\prime}$ is a $(a, b)$-swap of $P_{i}$. Further, for the other agent $j \neq i$, choose a preference $P_{j}$ such that $f\left(P_{i}, P_{j}\right)=b$. Since $a P_{i} b$, by Pareto efficiency, $b P_{j} a$. But $b P_{i}^{\prime} a$ implies $f\left(P_{i}^{\prime}, P_{j}\right) \neq a$. By swap monotonicity, $f\left(P_{i}^{\prime}, P_{j}\right)=b$. Hence, $f$ satisfies elementary monotonicity, and by Theorem 2, we are done.

\section{Proof of Theorem 3}

Let $f$ be a unanimous and G-LOBIC scf satisfying weak elementary monotonicity. The proof goes in many steps.

STEP 1. We start by stating a fact from Sato (2013).

FACT 1 (Sato (2013)) The single peaked domain is connected without restoration.

We now prove a claim using this fact.

Claim 1 Suppose $P_{i} \in \mathcal{S}$ is a preference ordering such that a $P_{i} b$. Then, there exists a preference ordering $P_{i}^{\prime} \in \mathcal{S}$ such that $P_{i}^{\prime}(1)=a$ and $B\left(b, P_{i}\right)=B\left(b, P_{i}^{\prime}\right)$. Moreover, for all $P_{-i} \in \mathcal{S}^{n-1}$ with $f\left(P_{i}, P_{-i}\right)=b$, we have $f\left(P_{i}^{\prime}, P_{-i}\right)=b$. 
Proof: The first part of the claim follows from the single peaked domain - if $a P_{i} b$, then we can always lift $a$ to the top and keep all the alternatives that are above $b$ in $P_{i}$ between $a$ and $b$ and all others below $b$ in the new preference ordering. Let $P_{i}^{\prime}$ be such an ordering. By Fact 1 , we know that there is a distinct sequence of preferences $\left(P_{i}=P^{0}, P^{1}, P^{2}, \ldots, P^{k}, P^{k+1}=P_{i}^{\prime}\right)$ without restoration such that consecutive preferences in the sequence are swaps of each other. Since $B\left(b, P_{i}\right)=B\left(b, P_{i}^{\prime}\right)$ and the sequence is without restoration, none of these swaps involve $b$. By repeatedly applying swap monotonicity along the sequence, we get $f\left(P_{i}^{\prime}, P_{-i}\right)=f\left(P_{i}, P_{-i}\right)=b$.

STEP 2. In this step, we show that if an scf is G-LOBIC and unanimous, then it must be Pareto efficient. For this consider a profile $\mathbf{P}$ with $f(\mathbf{P})=b$. Assume for contradiction that there exists $a \neq b$ such that $a P_{i} b$ for all $i \in N$. By repeated application of Claim 1 , there exists a preference profile $\mathbf{P}^{\prime}$ such that $f\left(\mathbf{P}^{\prime}\right)=b$ and $P_{i}^{\prime}(1)=a$ for all $i \in N$. This is a contradiction since unanimity implies that $f\left(\mathbf{P}^{\prime}\right)=a$.

SteP 3. Now, consider an agent $i \in N$ and $P_{-i} \in \mathcal{S}^{n-1}$. Let $P_{i}, P_{i}^{\prime} \in \mathcal{S}$ be such that $P_{i}^{\prime}$ is an $(a, b)$-swap of $P_{i}$ and $f\left(P_{i}, P_{-i}\right)=b$. We will show that $f\left(P_{i}^{\prime}, P_{-i}\right)=b$. This will show that $f$ satisfies elementary monotonicity and we will be done by Theorem 2 . Note that if agents in $P_{-i}$ have the same ranking of $a$ and $b$ then the claim is obvious. Hence, we assume that agents in $P_{-i}$ do not have the same ranking of $a$ and $b$. We consider two cases.

Case 3-1. Suppose $P_{i}(k)=a$ and $k>1$, i.e., the swap from $P_{i}$ to $P_{i}^{\prime}$ is not happening at the top of the preference ordering. Since $a$ and $b$ are consecutively ranked in $P_{i}$ and $P_{i}^{\prime}$ and neither of them are top ranked in $P_{i}$ and $P_{i}^{\prime}$, it must be that $a$ and $b$ are not neighbors (in $\succ)$. This is because if $a$ and $b$ are neighbors then they can only be swapped if they are at the top.

Hence, consider a neighbor $c$ of $a$ such that $c$ is between $a$ and $b$ in $\succ$ (i.e., if $a \succ b$, then $a \succ c, c \succ b$ and if $b \succ a$ then $b \succ c, c \succ a)$. By single peakedness, $c P_{i} a$ and $c P_{i}^{\prime} b$. Further, for any other agent $j \neq i$, there are four possible rankings between $a, b, c$ in $P_{j}$ : (1) $c P_{j} a P_{j} b$, (2) $c P_{j} b P_{j} a,(3) b P_{j} c P_{j} a$, and (4) $a P_{j} c P_{j} b$. The two profiles $\left(P_{i}, P_{-i}\right)$ and $\left(P_{i}^{\prime}, P_{-i}\right)$ are shown in Table 8 . Table 8 shows that there are four possible groups of agents in $P_{-i}$ with different rankings between $a, b, c$. We now modify the profile $\left(P_{i}, P_{-i}\right)$ in a sequence of steps to reach the profile $\left(P_{i}^{\prime}, P_{-i}\right)$.

STEP 3-1-A. In this step, we modify the preferences of agents in $P_{-i}$ who rank $a$ better than $c$ better than $b$. For each such agent $j$, we construct $P_{j}^{\prime}$ such that $P_{j}^{\prime}(1)=a, P_{j}^{\prime}(2)=c$ and $B\left(b, P_{j}^{\prime}\right)=B\left(b, P_{j}\right)$. Notice that since $a$ and $c$ are neighbors, single peakedness implies that 


\begin{tabular}{c|cccc||c|cccc}
$P_{i}$ & $P_{-i}$ & & & & $P_{i}^{\prime}$ & $P_{-i}$ & & & \\
\hline$\cdot$ & $\ldots$ & $\ldots$ & $\ldots$ & $\ldots$ & $\cdot$ & $\ldots$ & $\ldots$ & $\ldots$ & $\ldots$ \\
$c$ & $c \ldots$ & $c \ldots$ & $b \ldots$ & $a \ldots$ & $c$ & $c \ldots$ & $c \ldots$ & $b \ldots$ & $a \ldots$ \\
$\cdot$ & $\ldots$ & $\ldots$ & $\ldots$ & $\ldots$ & $\ldots$ & $\ldots$ & $\ldots$ & $\ldots$ & $\ldots$ \\
$a$ & $a \ldots$ & $b \ldots$ & $c \ldots$ & $c \ldots$ & $b$ & $a \ldots$ & $b \ldots$ & $c \ldots$ & $c \ldots$ \\
$b$ & $\ldots$ & $\ldots$ & $\ldots$ & $\ldots$ & $a$ & $\ldots$ & $\ldots$ & $\ldots$ & $\ldots$ \\
$\cdot$ & $b \ldots$ & $a \ldots$ & $a \ldots$ & $b \ldots$ &. & $b \ldots$ & $a \ldots$ & $a \ldots$ & $b \ldots$ \\
$\cdot$ & $\ldots$ & $\ldots$ & $\ldots$ & $\ldots$ &. & $\ldots$ & $\ldots$ & $\ldots$ & $\ldots$
\end{tabular}

Table 8: Profiles $\left(P_{i}, P_{-i}\right)$ and $\left(P_{i}^{\prime}, P_{-i}\right)$

such a $P_{j}^{\prime}$ can be constructed such that it is single peaked. Further, using a reasoning similar to Claim 1, we can argue that we can go from $P_{j}$ to $P_{j}^{\prime}$ using a without restoration sequence and since $B\left(b, P_{j}^{\prime}\right)=B\left(b, P_{j}\right), b$ will not be involved in any swaps. As a result, the outcome at the new profile will be $b$. The new profile is shown in Table 9 .

\begin{tabular}{c|cccc}
$P_{i}$ & Other & agents & & \\
\hline$\cdot$ & $\ldots$ & $\ldots$ & $\ldots$ & $a \ldots$ \\
$c$ & $c \ldots$ & $c \ldots$ & $b \ldots$ & $c \ldots$ \\
$\cdot$ & $\ldots$ & $\ldots$ & $\ldots$ & $\ldots$ \\
$a$ & $a \ldots$ & $b \ldots$ & $c \ldots$ & $\ldots$ \\
$b$ & $\ldots$ & $\ldots$ & $\ldots$ & $\ldots$ \\
$\cdot$ & $b \ldots$ & $a \ldots$ & $a \ldots$ & $b \ldots$ \\
$\cdot$ & $\ldots$ & $\ldots$ & $\ldots$ & $\ldots$
\end{tabular}

Table 9: Profile in Step 3-1-a.

SteP 3-1-B. In this step, we modify the profile in Table 9 as follows. For every agent, who ranks $a$ at the top, $c$ second, we perform the $(a, c)$-swap. Notice that this leads to a feasible single peaked preference ordering since $a$ and $c$ are neighbors. The new profile is shown in Table 10. By swap monotonicity, the outcome at the new profile is $b$.

SteP 3-1-c. In this step, we perform $(a, b)$-swap of $P_{i}$ to reach $P_{i}^{\prime}$. The new profile is shown in Table 11. By swap monotonicity, the outcome at the new profile is in $\{a, b\}$. Note that at the new profile $c P_{j} a$ for all $j \in N$. Hence, by Pareto efficiency the outcome at the new profile is $b$.

SteP 3-1-D. We can now consider the profile in Table 11 and alter the preferences of last 


\begin{tabular}{c|cccc}
$P_{i}$ & Other & agents & & \\
\hline$\cdot$ & $\ldots$ & $\ldots$ & $\ldots$ & $c \ldots$ \\
$c$ & $c \ldots$ & $c \ldots$ & $b \ldots$ & $a \ldots$ \\
. & $\ldots$ & $\ldots$ & $\ldots$ & $\ldots$ \\
$a$ & $a \ldots$ & $b \ldots$ & $c \ldots$ & $\ldots$ \\
$b$ & $\ldots$ & $\ldots$ & $\ldots$ & $\ldots$ \\
. & $b \ldots$ & $a \ldots$ & $a \ldots$ & $b \ldots$ \\
$\cdot$ & $\ldots$ & $\ldots$ & $\ldots$ & $\ldots$
\end{tabular}

Table 10: Profile in Step 3-1-b.

\begin{tabular}{c|cccc}
$P_{i}^{\prime}$ & Other & agents & & \\
\hline$\cdot$ & $\ldots$ & $\ldots$ & $\ldots$ & $c \ldots$ \\
$c$ & $c \ldots$ & $c \ldots$ & $b \ldots$ & $a \ldots$ \\
$\cdot$ & $\ldots$ & $\ldots$ & $\ldots$ & $\ldots$ \\
$b$ & $a \ldots$ & $b \ldots$ & $c \ldots$ & $\ldots$ \\
$a$ & $\ldots$ & $\ldots$ & $\ldots$ & $\ldots$ \\
$\cdot$ & $b \ldots$ & $a \ldots$ & $a \ldots$ & $b \ldots$ \\
$\cdot$ & $\ldots$ & $\ldots$ & $\ldots$ & $\ldots$
\end{tabular}

Table 11: Profile in Step 3-1-c.

(fifth) column of agents by performing a $(c, a)$-swap and then doing a sequence of without restoration swaps to go to their preference at the start of Step 3-1-A (see preferences in Table 8). The new profile is shown in Table 12.

\begin{tabular}{c|cccc}
$P_{i}^{\prime}$ & Other & agents & & \\
\hline$\cdot$ & $\ldots$ & $\ldots$ & $\ldots$ & $\ldots$ \\
$c$ & $c \ldots$ & $c \ldots$ & $b \ldots$ & $a \ldots$ \\
$\cdot$ & $\ldots$ & $\ldots$ & $\ldots$ & $\ldots$ \\
$b$ & $a \ldots$ & $b \ldots$ & $c \ldots$ & $c \ldots$ \\
$a$ & $\ldots$ & $\ldots$ & $\ldots$ & $\ldots$ \\
$\cdot$ & $b \ldots$ & $a \ldots$ & $a \ldots$ & $b \ldots$ \\
$\cdot$ & $\ldots$ & $\ldots$ & $\ldots$ & $\ldots$
\end{tabular}

Table 12: Profile in Step 3-1-d.

Since none of these swaps involve alternative $b$, the outcome at this new profile is $b$ due to swap monotonicity. But this profile is exactly $\left(P_{i}^{\prime}, P_{-i}\right)$. Hence, $f\left(P_{i}^{\prime}, P_{-i}\right)=b$. 
CASE 3-2. The other case is $P_{i}(k)=a$ and $k=1$, i.e., the swap from $P_{i}$ to $P_{i}^{\prime}$ is occurring at the top. The profile $\left(P_{i}, P_{-i}\right)$ is shown below in Table 13 . We now do the proof in many steps.

\begin{tabular}{c|cc}
$P_{i}$ & $P_{-i}$ & \\
\hline$a$ & $\ldots$ & $\ldots$ \\
$b$ & $a \ldots$ & $b \ldots$ \\
$\cdot$ & $\ldots$ & $\ldots$ \\
. & $b \ldots$ & $a \ldots$ \\
. & $\ldots$ & $\ldots$
\end{tabular}

Table 13: Profile in Case 3-2.

STEP 3-2-a. Now, consider every agent $j \neq i$ such that $a P_{j} b$ (agents in second column of Table 13). By Claim 1, we can construct a preference ordering from $P_{j}$ such that $a$ is top ranked and the outcome remains $b$. We change the preferences of all the agents in the profile of Table 13 who prefer $a$ to $b$ in this manner to arrive at the new profile. The new profile is shown in Table 14 and the outcome at the new profile is $b$.

\begin{tabular}{c|cc}
$P_{i}$ & Other & agents \\
\hline$a$ & $a \ldots$ & $\ldots$ \\
$b$ & $\ldots$ & $b \ldots$ \\
$\cdot$ & $\ldots$ & $\ldots$ \\
$\cdot$ & $b \ldots$ & $a \ldots$ \\
$\cdot$ & $\ldots$ & $\ldots$
\end{tabular}

Table 14: Profile in Step 3-2-a.

STEP 3-2-b. Now, for every agent $j \neq i$ such that $a$ is top tanked in the profile of Table 14, we consider a preference ordering where $a$ is top ranked and $b$ is second ranked. The new profile is shown in Table 15.

By Sato (2013), such a preference ordering can be reached by swaps without restoration. Hence, $a$ will not be involved in such swaps. Swap monotonicity implies that the outcome at the new profile is not $a$.

SteP 3-2-C. Now, consider every agent $j \neq i$ such that $b$ is preferred to $a$ in the preference profile in Step 3-2-b (agents in third column of Table 15). By Claim 1, we can construct a 


\begin{tabular}{c|cc}
$P_{i}$ & Other & agents \\
\hline$a$ & $a \ldots$ & $\ldots$ \\
$b$ & $b \ldots$ & $b \ldots$ \\
. & $\ldots$ & $\ldots$ \\
. & $\ldots$ & $a \ldots$ \\
. & $\ldots$ & $\ldots$
\end{tabular}

Table 15: Profile in Step 3-2-b.

preference ordering from this preference ordering such that $b$ is top ranked and the alternatives that were below $a$ do not change. The new profile is shown in Table 16. By definition, we can go to this new profile by doing a sequence of without restoration swaps that do not involve $a$. Hence, by swap monotonicity, the outcome at the new profile is not $a$. Then, by Pareto efficiency, the outcome at this new profile must be $b$.

\begin{tabular}{c|cc}
$P_{i}$ & Other & agents \\
\hline$a$ & $a \ldots$ & $b \ldots$ \\
$b$ & $b \ldots$ & $\ldots$ \\
$\cdot$ & $\ldots$ & $\ldots$ \\
. & $\ldots$ & $a \ldots$ \\
. & $\ldots$ & $\ldots$
\end{tabular}

Table 16: Profile in Step 3-2-c.

STEP 3-2-d. In this step, we consider all the agents who have $b$ top-ranked in the profile in Step 3-2-c (agents in the third column in Table 16). For every such agent, we consider another preference ordering where $b$ is top-ranked and $a$ is second ranked. The new profile is shown in Table 17. By Sato (2013), we can go to this new preference ordering by doing a sequence of without restoration swaps. Hence, by swap monotonicity, the outcome at the new profile is $b$.

Now, consider any agent who ranks $a$ at the top in the profile in Step 3-2-d. If his preference ordering is not the same as $P_{i}$, then we can transform it to $P_{i}$ by a sequence of without restoration swaps. Since this will not involve any swaps of $b$, by swap monotonicity the outcome will remain $b$ at the new profile. A similar argument can be made to transform the preference ordering of every agent who ranks $b$ at the top to the preference ordering $P_{i}^{\prime}$. As a consequence, the profile in Table 17 can be transformed to a profile where every agent $j \neq i$ has preference ordering $P_{i}$ or $P_{i}^{\prime}$ and the outcome at this profile is $b$. Denote this profile as $\left(P_{i}, \bar{P}_{-i}\right)$. This, we conclude that $f\left(P_{i}, \bar{P}_{-i}\right)=b$. 


\begin{tabular}{c|cc}
$P_{i}$ & Other & agents \\
\hline$a$ & $a \ldots$ & $b \ldots$ \\
$b$ & $b \ldots$ & $a \ldots$ \\
. & $\ldots$ & $\ldots$ \\
. & $\ldots$ & $\ldots$ \\
. & $\ldots$ & $\ldots$
\end{tabular}

Table 17: Profile in Step 3-2-d.

Now, assume for contradiction $f\left(P_{i}^{\prime}, P_{-i}\right)=a$. We now repeat the above procedure at the profile $\left(P_{i}^{\prime}, P_{-i}\right)$ to arrive at the profile $\left(P_{i}^{\prime}, \bar{P}_{-i}\right)$ and the outcome at this profile is $f\left(P_{i}^{\prime}, \bar{P}_{-i}\right)=a$. But $\left(P_{i}^{\prime}, \bar{P}_{-i}\right),\left(P_{i}, \bar{P}_{-i}\right) \in \mathcal{D}^{n}(2)$. Hence, $f\left(P_{i}, \bar{P}_{-i}\right)=b$ and $f\left(P_{i}^{\prime}, \bar{P}_{-i}\right)=a$ is a contradiction.

\section{Proof of Theorem 4}

Let $f$ be a tops-only, unanimous, G-LOBIC scf satisfying weak elementary monotonicity. We will show that $f$ satisfies elementary monotonicity and we will be done by Theorem 2 . Consider an agent $i \in N$ and a preference profile $\left(P_{i}, P_{-i}\right)$. Let $P_{i}^{\prime}$ be an $(a, b)$-swap of $P_{i}$ and $f\left(P_{i}, P_{-i}\right)=b$. By swap monotonicity, $f\left(P_{i}^{\prime}, P_{-i}\right) \in\{a, b\}$. Assume for contradiction that $f\left(P_{i}^{\prime}, P_{-i}\right)=a$. By tops-only property $P_{i}(2)=P_{i}^{\prime}(1)=b$ and $P_{i}(1)=P_{i}^{\prime}(2)=a$.

Notation: For any pair of adjacent preferences, $P$ and $P^{\prime}$, we say $P^{\prime}$ is a top-swap of $P$ if $P^{\prime}$ is a $(P(1), P(2))$-swap of $P$. We now do the proof in several steps, where each step achieves some technical milestone towards the eventual claim.

STEP 1. The profile $\left(P_{i}, P_{-i}\right)$ consists of agents who rank $a$ above $b$ and agents who rank $b$ above $a$ - see Table 18 .

Pick an agent $j \neq i$ such that $a P_{j} b$. Since the domain is connected without restoration, there exists a sequence of preferences without restoration $\left(P^{1}, \ldots, P^{k}\right)$ such that $P^{1} \equiv P_{j}$ and $P^{k} \equiv P_{i}$. By the without restoration property, for every $P^{\ell}$ in the sequence $a P^{\ell} b$, and hence, $P^{\ell}(1) \neq b$. This also implies that $b$ is never involved in a top-swap along the sequence. By tops-only property and swap monotonicity, we conclude that $f\left(P^{k}, P_{-j}\right)=b$. Repeating this argument for all agents $j \neq i$ such that $a P_{j} b$, we reach a profile shown in Table 19, where the outcome of $f$ is $b$.

SteP 2. Now, we consider an agent $j$ such that $b P_{j} a$. Since the domain is connected without restoration, there exists a sequence of preferences without restoration $\left(P^{1}, \ldots, P^{k}\right)$ such that 


\begin{tabular}{c|cc}
$P_{i}$ & $P_{-i}$ & \\
\hline$a$ & $\ldots$ & $\ldots$ \\
$b$ & $\ldots$ & $\ldots$ \\
$\cdot$ & $a \ldots$ & $b \ldots$ \\
. & $\ldots$ & $\ldots$ \\
$\cdot$ & $\ldots$ & $\ldots$ \\
$\cdot$ & $b \ldots$ & $a \ldots$ \\
. & $\ldots$ & $\ldots$ \\
. & $\ldots$ & $\ldots$
\end{tabular}

Table 18: Profile $\left(P_{i}, P_{-i}\right)$

\begin{tabular}{c|cc}
$P_{i}$ & Agents with preference $P_{i}$ & Other agents \\
\hline$a$ & $a \ldots$ & $\ldots$ \\
$b$ & $b \ldots$ & $\ldots$ \\
. & $\ldots$ & $b \ldots$ \\
. & $\ldots$ & $\ldots$ \\
. & $\ldots$ & $\ldots$ \\
. & $\ldots$ & $\ldots$ \\
. & $\ldots$ & $\ldots$ \\
. & $\ldots$ &
\end{tabular}

Table 19: Profile reached at the end of Step 1.

$P^{1} \equiv P_{j}$ and $P^{k} \equiv P_{i}^{\prime}$. Note that $P_{i}^{\prime}(1)=b$ and $P_{i}^{\prime}(2)=a$. Also, if $P_{j}(1)=b$, then $P_{j}=P_{i}^{\prime}$, and this sequence has exactly one preference. Else, this is a sequence without restoration, and $b$ will be involved in a top-swap at most once along the sequence. Let $P^{\ell}(1)=x, P^{\ell}(2)=b$ and $P^{\ell+1}(1)=b, P^{\ell+1}(2)=x$ for some $P^{\ell}$ along the sequence. Since this is a sequence without restoration, we conclude that $b$ is always ranked higher than $a$ along the sequence, and hence, $x \neq a$.

Now, for every $j$ such that $b P_{j} a$, we change the preference along the sequence to $P^{\ell}$. Since these changes do not involve top-swap of $b$, the outcome at the new profile is $b$. The new profile is shown in Table 20.

STEP 3. Now, we consider all the agents in the third column of the preference profile in Table 20 and change their preference to $P^{\ell+1}$. The new profile is shown in Table 21. By swap monotonicity the outcome of $f$ at this profile is either $x$ or $b$. We argue that it is $b$. Assume for contradiction that it is $x$. Then, we can change the preferences of agent $i$ and 


\begin{tabular}{c|cc}
$P_{i}$ & Agents with preference $P_{i}$ & Other agents \\
\hline$a$ & $a \ldots$ & $x \ldots$ \\
$b$ & $b \ldots$ & $b \ldots$ \\
. & $\ldots$ & $\ldots$ \\
. & $\ldots$ & $a \ldots$ \\
. & $\ldots$ & $\ldots$ \\
. & $\ldots$ & $\ldots$
\end{tabular}

Table 20: Profile at the end of Step 2.

all the agents in the second column of the preference profile in Table 21 to $P_{i}^{\prime}$ (note that all these agents have preference $P_{i}$ at the profile in Table 21). By swap monotonicity, the outcome of $f$ is at this new profile is still $x$, which will contradict unanimity.

\begin{tabular}{c|cc}
$P_{i}$ & $\hat{P}_{-i}$ & \\
\hline$a$ & $a \ldots$ & $b \ldots$ \\
$b$ & $b \ldots$ & $x \ldots$ \\
$\cdot$ & $\ldots$ & $\ldots$ \\
. & $\ldots$ & $a \ldots$ \\
$\cdot$ & $\ldots$ & $\ldots$ \\
. & $\ldots$ & $\ldots$
\end{tabular}

Table 21: Profile in Step

STEP 4. Finally, we can change the preference of all the agents in the third column of the profile in Table 21 along the sequence $\left(P^{\ell+1}, \ldots, P^{k}\right)$, where $P^{k}=P_{i}^{\prime}$. The new profile is shown in Table 22. Since no swaps along this sequence involves $b$, swap monotonicity ensures that the outcome at this top- 2 profile is $b$. Denote this preference profile as $\left(P_{i}, \hat{P}_{-i}\right)$. An analogous argument starting from the profile $\left(P_{i}^{\prime}, P_{-i}\right)$ and ending at $\left(P_{i}^{\prime}, \hat{P}_{-i}\right)$ can be made to show that $f\left(P_{i}^{\prime}, \hat{P}_{-i}\right)=a$. But $f\left(P_{i}, \hat{P}_{-i}\right)=b$ contradicts weak elementary monotonicity.

\section{Proof of Lemma 1}

The scf $\bar{f}$ is clearly unanimous. To see that it satisfies weak elementary monotonicity, consider the top-2 profiles when (a) a non-empty subset of agents have preference $P^{1}$ and the remaining non-empty subset of agents have preference $P^{2}$ and (b) a non-empty subset of agents have preference $P^{5}$ and the remaining non-empty set of agents have preference $P^{6}$. 


\begin{tabular}{c|cc}
$P_{i}$ & Agents with preference $P_{i}$ & Agents with preference $P_{i}^{\prime}$ \\
\hline$a$ & $a \ldots$ & $b \ldots$ \\
$b$ & $b \ldots$ & $a \ldots$ \\
. & $\ldots$ & $\ldots$ \\
. & $\ldots$ & $\ldots$
\end{tabular}

Table 22: Profile in Step - A top-2 profile

In both the cases, the outcome of $\bar{f}$ at such a profile cannot be $b$ and if it is $a$ or $d$, then it clearly continues to remain the same across the two profiles (this is because relative ranking of $a$ and $d$ for any agent does not change across these profiles).

Thus, $\bar{f}$ satisfies weak elementary monotonicity.

To see that $\bar{f}$ does not satisfy tops-onlyness, note that $f\left(P_{1}, P_{3}, P_{4}\right)=d$ but $f\left(P_{1}, P_{4}, P_{4}\right)=$ $a$ and the tops of the agents at these two profiles do not change. Since $a P_{3} d$, this also shows that agent 2 can manipulate at profile $\left(P_{1}, P_{3}, P_{4}\right)$ by reporting $P_{4}$. Hence, $\bar{f}$ is not DSIC.

Finally, we show that $\bar{f}$ is G-LOBIC. Fix any generic prior $\left\{\mu_{i}\right\}_{i \in N}$. Denote the domain of preferences given in Table 4 as $\mathcal{D}$. For notational simplicity, we will denote by $q_{i}^{\ell} \equiv \mu_{i}\left(P^{\ell}\right)$ the probability that agent $i \in\{1,2,3\}$ has preference ordering $P^{\ell}$ for every $P^{\ell} \in \mathcal{D}$. Further, for every $i \in N$, we denote by $q_{i}^{123} \equiv q_{i}^{1}+q_{i}^{2}+q_{i}^{3}, q_{i}^{23} \equiv q_{i}^{2}+q_{i}^{3}, q_{i}^{45} \equiv q_{i}^{4}+q_{i}^{5}$, and $q_{i}^{2345} \equiv q_{i}^{23}+q_{i}^{45}$.

We will compute the interim outcome probability of $\bar{f}$ using these priors. We consider various cases. We denote an arbitrary agent as $i \in\{1,2,3\}$ and the other two agents as $j$ and $k$.

CASE $1-P^{1}$. If agent $i$ has preference $P^{1}$, then the outcome in $\bar{f}$ can be only $a$ and $d$. The outcome is $a$ if both $j$ and $k$ have preference in $\left\{P^{1}, P^{2}, P^{3}\right\}$ or both have their preference in $\left\{P^{4}, P^{5}, P^{6}, P^{7}\right\}$. This probability is given by

$$
\begin{aligned}
\pi_{i}^{\bar{f}}\left(a, P^{1}\right) & =q_{j}^{123} q_{k}^{123}+\left(1-q_{j}^{123}\right)\left(1-q_{k}^{123}\right) \\
& =1-q_{j}^{123}\left(1-q_{k}^{123}\right)-q_{k}^{123}\left(1-q_{j}^{123}\right) .
\end{aligned}
$$

From this we can compute $\pi_{i}^{\bar{f}}\left(d, P^{1}\right)$ as

$$
\pi_{i}^{\bar{f}}\left(d, P^{1}\right)=q_{j}^{123}\left(1-q_{k}^{123}\right)+q_{k}^{123}\left(1-q_{j}^{123}\right) .
$$

CASE $2-P^{2}, P^{3}$. If preference of agent $i$ is $P^{2}$ or $P^{3}$, then $\bar{f}$ can only choose $b$ or $d$ or $a$. Alternative $b$ is chosen if both $j$ and $k$ have $b$ at the top, i.e., both have preference in $\left\{P^{2}, P^{3}, P^{4}, P^{5}\right\}$. Hence, we can write

$$
\pi_{i}^{\bar{f}}\left(b, P^{2}\right)=\pi_{i}^{\bar{f}}\left(b, P^{3}\right)=q_{j}^{2345} q_{k}^{2345}
$$


Alternative $d$ is chosen if exactly two agents prefer $a$ to $d$. This happens if exactly one agent besides $i$ has preference in $\left\{P^{1}, P^{2}, P^{3}\right\}$ and the other agent has preference outside $\left\{P^{1}, P^{2}, P^{3}\right\}$ but the preferences of both these agents should not lie in $\left\{P^{2}, P^{3}, P^{4}, P^{5}\right\}$. The probability of this event is

$$
\pi_{i}^{\bar{f}}\left(d, P^{2}\right)=\pi_{i}^{\bar{f}}\left(d, P^{3}\right)=q_{j}^{123}\left(1-q_{k}^{123}\right)+q_{k}^{123}\left(1-q_{j}^{123}\right)-q_{j}^{23} q_{k}^{45}-q_{k}^{23} q_{j}^{45} .
$$

This also means that the interim outcome probability of alternative $a$ is given by

$$
\pi_{i}^{\bar{f}}\left(a, P^{2}\right)=\pi_{i}^{\bar{f}}\left(a, P^{3}\right)=1-q_{j}^{123}\left(1-q_{k}^{123}\right)-q_{k}^{123}\left(1-q_{j}^{123}\right)+q_{j}^{23} q_{k}^{45}+q_{k}^{23} q_{j}^{45}-q_{j}^{2345} q_{k}^{2345} .
$$

CAse $3-P^{4}, P^{5}$. This case is similar to Case 2 with interim outcome probabilities of $a$ and $d$ switched.

$$
\begin{aligned}
& \pi_{i}^{\bar{f}}\left(b, P^{4}\right)=\pi_{i}^{\bar{f}}\left(b, P^{5}\right)=q_{j}^{2345} q_{k}^{2345} \\
& \pi_{i}^{\bar{f}}\left(a, P^{4}\right)=\pi_{i}^{\bar{f}}\left(a, P^{5}\right)=q_{j}^{123}\left(1-q_{k}^{123}\right)+q_{k}^{123}\left(1-q_{j}^{123}\right)-q_{j}^{23} q_{k}^{45}-q_{k}^{23} q_{j}^{45} . \\
& \pi_{i}^{\bar{f}}\left(d, P^{4}\right)=\pi_{i}^{\bar{f}}\left(d, P^{5}\right)=1-q_{j}^{123}\left(1-q_{k}^{123}\right)-q_{k}^{123}\left(1-q_{j}^{123}\right)+q_{j}^{23} q_{k}^{45}+q_{k}^{23} q_{j}^{45}-q_{j}^{2345} q_{k}^{2345} .
\end{aligned}
$$

CASE $4-P^{6}, P^{7}$. In this case, only $d$ and $a$ can be outcome of the scf. Alternative $a$ is the outcome if exactly one of the agents in $\{j, k\}$ has preference in $\left\{P^{1}, P^{2}, P^{3}\right\}$ but the other agent has preference outside $\left\{P^{1}, P^{2}, P^{3}\right\}$. The probability of this event is

$$
\pi^{\bar{f}}\left(a, P^{6}\right)=\pi^{\bar{f}}\left(d, P^{7}\right)=q_{j}^{123}\left(1-q_{k}^{123}\right)+q_{k}^{123}\left(1-q_{j}^{123}\right)
$$

Hence, the interim outcome probability of alternative $d$ is

$$
\pi^{\bar{f}}\left(d, P^{6}\right)=\pi^{\bar{f}}\left(a, P^{7}\right)=1-q_{j}^{123}\left(1-q_{k}^{123}\right)-q_{k}^{123}\left(1-q_{j}^{123}\right)
$$

Now, we find conditions on priors that guarantee $\bar{f}$ is G-LOBIC. From the calculations above, we can do this by considering the following cases about the possible true preference orderings of agent $i$.

CASE $1-P^{1}$. In this case, agent $i$ does not manipulate if $\pi_{i}^{\bar{f}}\left(a, P^{1}\right) \geq \pi_{i}^{\bar{f}}\left(a, P^{j}\right)$ for any $P^{j} \in \mathcal{D}$. Note that $\pi_{i}^{\bar{f}}\left(a, P^{1}\right)>\pi_{i}^{\bar{f}}\left(a, P^{2}\right)=\pi_{i}^{\bar{f}}\left(a, P^{3}\right)$ since $q_{j}^{2345} q_{k}^{2345}>q_{j}^{23} q_{k}^{45}+q_{j}^{45} q_{k}^{23}$. Now, $\pi_{i}^{\bar{f}}\left(a, P^{1}\right) \geq \pi_{i}^{\bar{f}}\left(a, P^{4}\right)=\pi_{i}^{\bar{f}}\left(a, P^{5}\right)$ if and only if

$$
q_{j}^{123}\left(1-q_{k}^{123}\right)+q_{k}^{123}\left(1-q_{j}^{123}\right) \leq \frac{1}{2}+\frac{1}{2}\left[q_{j}^{23} q_{k}^{45}+q_{j}^{45} q_{k}^{23}\right]
$$

Note that this can be generically satisfied by choosing values of $q_{j}^{123}$ and $q_{k}^{123}$ close to zero. Finally, we need $\pi_{i}^{\bar{f}}\left(a, P^{1}\right) \geq \pi_{i}^{\bar{f}}\left(a, P^{6}\right)=\pi_{i}^{\bar{f}}\left(a, P^{7}\right)$. This is possible if and only if

$$
q_{j}^{123}\left(1-q_{k}^{123}\right)+q_{k}^{123}\left(1-q_{j}^{123}\right) \leq \frac{1}{2} .
$$


Note that this can be generically satisfied by choosing values of $q_{j}^{123}$ and $q_{k}^{123}$ close to zero.

CASE $2-P^{2}, P^{3}$. In this case, note that $\pi_{i}^{\bar{f}}\left(b, P^{2}\right)=\pi_{i}^{\bar{f}}\left(b, P^{3}\right) \geq \pi_{i}^{\bar{f}}\left(b, P^{j}\right)$ for all $j$. Hence, G-OBIC only requires the following inequality to hold for all $j$ :

$$
\pi_{i}^{\bar{f}}\left(b, P^{2}\right)+\pi_{i}^{\bar{f}}\left(a, P^{2}\right)=\pi_{i}^{\bar{f}}\left(b, P^{3}\right)+\pi_{i}^{\bar{f}}\left(a, P^{3}\right) \geq \pi_{i}^{\bar{f}}\left(b, P^{j}\right)+\pi_{i}^{\bar{f}}\left(a, P^{j}\right)
$$

Clearly this is true if $P^{j}=P^{1}$. If $P^{j} \in\left\{P^{4}, P^{5}\right\}$, then the above inequality holds if and only if

$$
q_{j}^{123}\left(1-q_{k}^{123}\right)+q_{k}^{123}\left(1-q_{j}^{123}\right) \leq \frac{1}{2}+q_{j}^{23} q_{k}^{45}+q_{j}^{45} q_{k}^{23}-\frac{1}{2} q_{j}^{2345} q_{k}^{2345} .
$$

This can be satisfied generically if we pick values of $q_{j}^{\ell}$ for all $\ell \in\{1,2,3,4,5\}$ close to zero. As a result, the RHS will be close to $\frac{1}{2}$ and the LHS will be close to zero. Finally, if $P^{j} \in\left\{P^{6}, P^{7}\right\}$, G-OBIC holds if and only if

$$
q_{j}^{123}\left(1-q_{k}^{123}\right)+q_{k}^{123}\left(1-q_{j}^{123}\right) \leq \frac{1}{2}+\frac{1}{2}\left(q_{j}^{23} q_{k}^{45}+q_{j}^{45} q_{k}^{23}\right)
$$

This can be satisfied generically if we pick (as in Case 1 ) values of $q_{j}^{123}$ and $q_{k}^{123}$ close to zero.

CASE $3-P^{4}, P^{5}$. Like in Case $2, \pi_{i}^{\bar{f}}\left(b, P^{4}\right)=\pi_{i}^{\bar{f}}\left(b, P^{5}\right) \geq \pi_{i}^{\bar{f}}\left(b, P^{j}\right)$ for all $j$. Hence, G-OBIC only requires the following inequality to hold for all $j$ :

$$
\pi_{i}^{\bar{f}}\left(b, P^{4}\right)+\pi_{i}^{\bar{f}}\left(d, P^{4}\right)=\pi_{i}^{\bar{f}}\left(b, P^{5}\right)+\pi_{i}^{\bar{f}}\left(d, P^{5}\right) \geq \pi_{i}^{\bar{f}}\left(b, P^{j}\right)+\pi_{i}^{\bar{f}}\left(d, P^{j}\right) .
$$

Clearly, this is true if $P^{j} \in\left\{P^{6}, P^{7}\right\}$. If $P^{j}=P^{1}$, then this is equivalent to requiring

$$
q_{j}^{123}\left(1-q_{k}^{123}\right)+q_{k}^{123}\left(1-q_{j}^{123}\right) \leq \frac{1}{2}+\frac{1}{2}\left[q_{j}^{23} q_{k}^{45}+q_{j}^{45} q_{k}^{23}\right]
$$

This can be generically satisfied by picking values of $q_{j}^{123}$ and $q_{k}^{123}$ close to zero. If $P^{j} \in$ $\left\{P^{2}, P^{3}\right\}$, the G-OBIC inequality is equivalent to requiring

$$
q_{j}^{123}\left(1-q_{k}^{123}\right)+q_{k}^{123}\left(1-q_{j}^{123}\right) \leq \frac{1}{2}+q_{j}^{23} q_{k}^{45}+q_{j}^{45} q_{k}^{23}-\frac{1}{2} q_{j}^{2345} q_{k}^{2345} \text {. }
$$

This can be satisfied generically if we pick values of $q_{j}^{\ell}$ close to zero for all $\ell \in\{1,2,3,4,5\}$.

CASE $4-P^{6}, P^{7}$. In this case, agent $i$ does not manipulate if $\pi_{i}^{\bar{f}}\left(d, P^{1}\right) \geq \pi_{i}^{\bar{f}}\left(d, P^{j}\right)$ for any $P^{j} \in \mathcal{D}$. This is clearly true if $P^{j} \in\left\{P^{4}, P^{5}\right\}$. If $P^{j}=P^{1}$, then this is equivalent to requiring

$$
q_{j}^{123}\left(1-q_{k}^{123}\right)+q_{k}^{123}\left(1-q_{j}^{123}\right) \leq \frac{1}{2}
$$


which can be satisfied generically by choosing $q_{j}^{\ell}$ close to zero for all $\ell \in\{1,2,3\}$. If $P^{j} \in$ $\left\{P^{2}, P^{3}\right\}$, this is equivalent to requiring

$$
q_{j}^{123}\left(1-q_{k}^{123}\right)+q_{k}^{123}\left(1-q_{j}^{123}\right) \leq \frac{1}{2}+\frac{1}{2}\left[q_{j}^{23} q_{k}^{45}+q_{j}^{45} q_{k}^{23}\right]
$$

which can be satisfied generically by choosing $q_{j}^{\ell}$ close to zero for all $\ell \in\{1,2,3\}$.

This completes all the cases. Summarizing, if the probabilities of the preferences in $\left\{P^{1}, \ldots, P^{5}\right\}$ are small enough and the remaining probabilities are high enough, then OBIC holds for $\bar{f}$. Since this can be done generically $\bar{f}$ is G-OBIC.

\section{Proof of Theorem 5}

We first show that if $f: \mathcal{P}^{n} \rightarrow A$ is G-LOBIC and unanimous, then it is Pareto efficient. Suppose $f$ is G-LOBIC and unanimous but assume for contradiction that it is not Pareto efficient. For this, we consider a profile $\mathbf{P}$ such that $f(\mathbf{P})=b$ but there exists $a \in A$ such that $a P_{i} b$ for all $i \in N$. Consider an agent $i \in N$ such that $P_{i}(k)=a$ and $k \neq 1$. Suppose $P_{i}(k-1)=x$. Consider $P_{i}^{\prime}$ which is a $(x, a)$-swap of $P_{i}$. By swap monotonicity, $f\left(P_{i}^{\prime}, P_{-i}\right)=b$. We can repeat such swaps to reach a preference ordering $P_{i}^{\prime \prime}$ for agent $i$ such that $P_{i}^{\prime \prime}(1)=a$ and $f\left(P_{i}^{\prime \prime}, P_{-i}\right)=b$. Now, we can repeat this procedure for every agent $j$ such that $P_{j}(k)=a$ and $k \neq 1$ to arrive at a profile $\mathbf{P}^{\prime \prime}$ such that $f\left(\mathbf{P}^{\prime \prime}\right)=b$. But this will contradict unanimity since $P_{j}^{\prime \prime}(1)=a$ for all $j \in N$.

Hence, we show that any $f: \mathcal{P}^{n} \rightarrow A$ that is G-LOBIC and Pareto efficient must satisfy elementary monotonicity. By Theorem 2, we will be done.

To do so, we consider an agent $i \in N$, a preference profile $P_{-i}$ of other agents, and $P_{i}, P_{i}^{\prime}$ such that $P_{i}^{\prime}$ is an $(a, b)$-swap of $P_{i}$ and $f\left(P_{i}, P_{-i}\right)=b$. The two profiles are shown in Table 23. Notice that there are some agents in $P_{-i}$ who prefer $a$ to $b$ and some prefer $b$ to $a$.

\begin{tabular}{c|cc||c|cc}
$P_{i}$ & $P_{-i}$ & & $P_{i}^{\prime}$ & $P_{-i}$ & \\
\hline$\cdot$ & $\ldots$ & $\ldots$ & $\cdot$ & $\ldots$ & $\ldots$ \\
$\cdot$ & $\ldots$ & $\ldots$ & $\cdot$ & $\ldots$ & $\ldots$ \\
$a$ & $b \ldots$ & $a \ldots$ & $b$ & $b \ldots$ & $a \ldots$ \\
$b$ & $\ldots$ & $\ldots$ & $a$ & $\ldots$ & $\ldots$ \\
$\cdot$ & $a \ldots$ & $b \ldots$ & $\cdot$ & $a \ldots$ & $b \ldots$ \\
$\cdot$ & $\ldots$ & $\ldots$ & $\cdot$ & $\ldots$ & $\ldots$
\end{tabular}

Table 23: Profiles $\left(P_{i}, P_{-i}\right)$ and $\left(P_{i}^{\prime}, P_{-i}\right)$

We will now show that $f\left(P_{i}^{\prime}, P_{-i}\right)=b$. By swap monotonicity, $f\left(P_{i}^{\prime}, P_{-i}\right) \in\{a, b\}$. Assume for contradiction that $f\left(P_{i}^{\prime}, P_{-i}\right)=a$. Now, we do the proof in several steps. 
STEP 1. We modify the profile $\left(P_{i}, P_{-i}\right)$ to bring one of the alternatives not in $\{a, b\}$ (such an alternative exists since $|A| \geq 3$ ) just below $\{a, b\}$ for all the agents. Let $x \notin\{a, b\}$ be some alternative. If $a P_{j} x$ and $b P_{j} x$ for some $j \in N$, then we can do a series of swaps to lift $x$ up such that it is just below $b$ if $a P_{j} b$ or just below $a$ if $b P_{j} a$ (note that none of these swaps will involve $b$ ). By swap monotonicity, the outcome at the new profile continues to be $b$. Using a similar argument, if $b P_{j} x$ and $x P_{j} a$ for some $j \in N$, then we can come to a preference ordering where $x$ is just below a maintaining the outcome to be $b$.

Now, consider $j \in N$, such that $x P_{j} b$. If $x$ and $b$ are not consecutive in $P_{j}$, then again we can do a series of swaps to come to a preference ordering such that $x$ is just above $b$ (note again that none of these swaps will involve $b$ ). By swap monotonicity, the outcome at the new profile continues to be $b$. Let us denote this new profile by $\overline{\mathbf{P}}$.

\begin{tabular}{cccc}
$\overline{\mathbf{P}}$ & & & \\
\hline$\ldots$ & $\ldots$ & $\ldots$ & $\ldots$ \\
$\ldots$ & $\ldots$ & $\ldots$ & $\ldots$ \\
$a$ & $b$ & $a$ & $x$ \\
$\ldots$ & $\ldots$ & $\ldots$ & $b$ \\
$\ldots$ & $\ldots$ & $\ldots$ & $\ldots$ \\
$b$ & $a$ & $x$ & $\ldots$ \\
$x$ & $x$ & $b$ & $a$ \\
$\ldots$ & $\ldots$ & $\ldots$ & $\ldots$ \\
$\ldots$ & $\ldots$ & $\ldots$ & $\ldots$
\end{tabular}

Table 24: Profile $\overline{\mathbf{P}}$

So, we have reached a profile $\overline{\mathbf{P}}$, where for every $j \in N$, either $x$ is just above $b$ in $\bar{P}_{j}$ or [ $x$ is just below $b$ if $a \bar{P}_{j} b$ and $x$ is just below $a$ if $b \bar{P}_{j} a$ ]. Table 24 shows the profile $\overline{\mathbf{P}}$.

Now, for every $j \in N$ such that $x$ is just above $b$ in $\bar{P}_{j}$ (Columns 3 and 4 in Table 24). We do a $(x, b)$-swap. By swap monotonicity the outcome at the new profile is either $x$ or $b$. But $b$ is preferred to $x$ by all the agents, and hence, Pareto efficiency implies that the outcome at this profile is $b$. For every $j$ belonging to Column 4 in Table 24, we then do a sequence of swaps to get $x$ just below $a$. Denote this new preference ordering by $\bar{P}_{j}^{\prime}$. By swap monotonicity the outcome at the new profile is $b$. Denote the new profile as $\overline{\mathbf{P}}^{\prime}$ and note that $f\left(\overline{\mathbf{P}}^{\prime}\right)=b$.

Now, consider the $(a, b)$-swap of $\bar{P}_{i}^{\prime}$ and denote this preference ordering as $\bar{P}_{i}^{\prime \prime}$. Since $f\left(P_{i}^{\prime}, P_{-i}\right)=a$, an analogous argument will show that $f\left(\bar{P}_{i}^{\prime \prime}, \bar{P}_{-i}^{\prime}\right)=a$. The two profiles $\left(\bar{P}_{i}^{\prime}, \bar{P}_{-i}^{\prime}\right)$ and $\left(\bar{P}_{i}^{\prime \prime}, \bar{P}_{-i}^{\prime}\right)$ are shown in Table 25. 


\begin{tabular}{c|cc||c|cc}
$\bar{P}_{i}^{\prime}$ & $\bar{P}_{-i}^{\prime}$ & & $\bar{P}_{i}^{\prime \prime}$ & $\bar{P}_{-i}^{\prime}$ & \\
\hline$\cdot$ & $\ldots$ & $\ldots$ & $\cdot$ & $\ldots$ & $\ldots$ \\
$\cdot$ & $\ldots$ & $\ldots$ & $\cdot$ & $\ldots$ & $\ldots$ \\
$a$ & $b \ldots$ & $a \ldots$ & $b$ & $b \ldots$ & $a \ldots$ \\
$b$ & $\ldots$ & $\ldots$ & $a$ & $\ldots$ & $\ldots$ \\
$x$ & $a \ldots$ & $b \ldots$ & $x$ & $a \ldots$ & $b \ldots$ \\
$\cdot$ & $x \ldots$ & $x \ldots$ & $\cdot$ & $x \ldots$ & $x \ldots$ \\
$\cdot$ & $\ldots$ & $\ldots$ & $\cdot$ & $\ldots$ & $\ldots$
\end{tabular}

Table 25: Profiles $\left(\bar{P}_{i}^{\prime}, \bar{P}_{-i}^{\prime}\right)$ and $\left(\bar{P}_{i}^{\prime \prime}, \bar{P}_{-i}^{\prime}\right)$

STEP 2. In this step, we modify the profile $\left(\bar{P}_{i}^{\prime}, \bar{P}_{-i}^{\prime}\right)$ in a particular way. First, we look at an agent $j \in N$, such that $a \bar{P}_{j}^{\prime} b$ and $b \bar{P}_{j}^{\prime} x$. We perform a $(b, x)$ swap for each of these agents. The new profile is shown in Table 26. By swap monotonicity, the outcome at the new profile must be in $\{b, x\}$. But since $a$ is ranked higher than $x$ for all the agents, Pareto efficiency implies the outcome at the new profile must be $b$.

\begin{tabular}{c|cc|} 
Agent $i$ & Other agents & \\
\hline$\cdot$ & $\ldots$ & $\ldots$ \\
$\cdot$ & $\ldots$ & $\ldots$ \\
$a$ & $b \ldots$ & $a \ldots$ \\
$x$ & $\ldots$ & $\ldots$ \\
$b$ & $a \ldots$ & $x \ldots$ \\
. & $x \ldots$ & $b \ldots$ \\
. & $\ldots$ & $\ldots$
\end{tabular}

Table 26: New profile in Step 2

STEP 3. In this step, we modify the profile in Table 26 further. In particular, we lift $x$ just above $a$. For agent $i$ and for all $j \neq i$ such that $x$ is just below $a$, this can be done by a $(a, x)$-swap. For all other agents, this requires a series of swaps - note that these swaps can be done by without involving $b$. The new profile is shown in Table 27 . Since none of the swaps involve $b$, swap monotonicity implies that the outcome at the new profile remains $b$.

STEP 4. In this step, we modify the profile in Step 3 by changing only agent $i$ 's preference ordering. We do this by doing an $(a, b)$-swap of the preference ordering of agent $i$ in the profile shown in Table 27. The new profile is shown in Table 28. By swap monotonicity, the outcome at the new profile is in $\{a, b\}$. But $x$ is better than $a$ for all agents, and hence, 


\begin{tabular}{c|cc|} 
Agent $i$ & Other agents & \\
\hline$\cdot$ & $\ldots$ & $\ldots$ \\
. & $\ldots$ & $\ldots$ \\
$x$ & $b \ldots$ & $x \ldots$ \\
$a$ & $\ldots$ & $a \ldots$ \\
$b$ & $x \ldots$ & $\ldots$ \\
. & $a \ldots$ & $b \ldots$ \\
. & $\ldots$ & $\ldots$
\end{tabular}

Table 27: New profile in Step 3

Pareto efficiency implies the outcome at the new profile is $b$.

\begin{tabular}{c|cc|} 
Agent $i$ & Other agents & \\
\hline$\cdot$ & $\ldots$ & $\ldots$ \\
$\cdot$ & $\ldots$ & $\ldots$ \\
$x$ & $b \ldots$ & $x \ldots$ \\
$b$ & $\ldots$ & $a \ldots$ \\
$a$ & $x \ldots$ & $\ldots$ \\
$\cdot$ & $a \ldots$ & $b \ldots$ \\
$\cdot$ & $\ldots$ & $\ldots$
\end{tabular}

Table 28: New profile in Step 4

STEP 5. In this step, we modify the profile in Step 4 by changing the preferences of those agents who prefer $x$ to $a$ and $a$ to $b$ (the third column of agents in Table 28). We perform a series of swaps to bring $x$ just one position above $b$. The new profile is shown in Table 29 . By swap monotonicity, the outcome at this profile remains $b$.

STEP 6. Now, we perform an $(x, b)$-swap of preferences of those agents who rank $x$ just above $b$ in the profile in Step 5 - this will be agent $i$ and agents in the third column in Table 29. The new profile is shown in Table 30. By swap monotonicity, the outcome at the new profile is in $\{x, b\}$. But $b$ is preferred to $x$ for all the agents. Hence, Pareto efficiency implies the outcome at the new profile remains $b$.

STEP 7. Finally, we perform a $(x, a)$-swap for the preferences of all agents in the profile in Step 6 who rank $x$ just above $a$ - this will include agent $i$ and agents in the second column of Table 30. The new profile is shown Table 31. By swap monotonicity, the outcome at this 


\begin{tabular}{c|cc|} 
Agent $i$ & Other agents & \\
\hline$\cdot$ & $\ldots$ & $\ldots$ \\
$\cdot$ & $\ldots$ & $\ldots$ \\
$x$ & $b \ldots$ & $a \ldots$ \\
$b$ & $\ldots$ & $\ldots$ \\
$a$ & $x \ldots$ & $x \ldots$ \\
$\cdot$ & $a \ldots$ & $b \ldots$ \\
$\cdot$ & $\ldots$ & $\ldots$
\end{tabular}

Table 29: New profile in Step 5

\begin{tabular}{c|cc|} 
Agent $i$ & Other agents & \\
\hline$\cdot$ & $\ldots$ & $\ldots$ \\
$\cdot$ & $\ldots$ & $\ldots$ \\
$b$ & $b \ldots$ & $a \ldots$ \\
$x$ & $\ldots$ & $\ldots$ \\
$a$ & $x \ldots$ & $b \ldots$ \\
$\cdot$ & $a \ldots$ & $x \ldots$ \\
$\cdot$ & $\ldots$ & $\ldots$
\end{tabular}

Table 30: New profile in Step 6

profile remains $b$.

But the profile shown in Table 31 is exactly the profile $\left(\bar{P}_{i}^{\prime \prime}, \bar{P}_{-i}^{\prime}\right)$ (see Table 25) and we had assumed that $f\left(\bar{P}_{i}^{\prime \prime}, \bar{P}_{-i}^{\prime}\right)=a$. This is a contradiction.

\begin{tabular}{c|cc|} 
Agent $i$ & Other agents & \\
\hline$\cdot$ & $\ldots$ & $\ldots$ \\
$\cdot$ & $\ldots$ & $\ldots$ \\
$b$ & $b \ldots$ & $a \ldots$ \\
$a$ & $\ldots$ & $\ldots$ \\
$x$ & $a \ldots$ & $b \ldots$ \\
$\cdot$ & $x \ldots$ & $x \ldots$ \\
$\cdot$ & $\ldots$ & $\ldots$
\end{tabular}

Table 31: New profile in Step 7 


\section{REFERENCES}

BarberÀ, S., D. Berga, And B. Moreno (2012): "Domains, ranges and strategyproofness: the case of single-dipped preferences," Social Choice and Welfare, 39, 335-352.

Bhargava, M., D. Majumdar, and A. Sen (2014): "Incentive-Compatible Voting Rules with Positively Correlated Beliefs," Forthcoming, Theoretical Economics.

Carroll, G. (2012): "When are local incentive constraints sufficient?" Econometrica, 80, 661-686.

Chambers, C. And A. Miller (2011): "Rules for Aggregating Information," Social Choice and Welfare, 36, 75-82.

Chatterji, S., R. Sanver, And A. Sen (2013): "On Domains that Admit Well-Behaved Strategy-proof Social Choice Functions," Journal of Economic Theory, 148.

ChatterJi, S. And A. Sen (2011): "Tops-only domains," Economic Theory, 46, 255-282.

D’Aspremont, C. And B. Peleg (1988): "Ordinal Bayesian Incentive Compatible Representations of Committees," Social Choice and Welfare, 5, 261-279.

Demange, G. (1982): "Single-peaked Orders on a Tree," Mathematical Social Sciences, 3, 389-396.

EhLers, L. AND J. MAsso (2007): "Incomplete Information and Singleton Cores in Matching Markets," Journal of Economic Theory, 136, 587-600.

(2015): "Matching markets under (in)complete information," Journal of Economic Theory, 157, 295-314.

Fishburn, P. C. And A. Rubinstein (1986): "Aggregation of Equivalence Relations," Journal of Classification, 3, 61-65.

Gershkov, A., J. K. Goeree, A. Kushnir, B. Moldovanu, and X. Shi (2013): "On the Equivalence of Bayesian and Dominant Strategy Implementation," Econometrica, 81, $197-220$.

GibBard, A. (1973): "Manipulation of Voting Schemes: A General Result," Econometrica, $41,587-602$.

Kalai, E. And U. Muller (1977): "Characterization of Domains Admitting Nondictatorial Social Welfare Functions and Nonmanipulable Voting Procedures*," Journal of Economic Theory, 16, 457-469. 
Klaus, B., H. Peters, And T. Storcken (1997): "Strategy-proof division of a private good when preferences are single-dipped," Economics Letters, 55, 339-346.

Majumdar, D. (2003): "Ordinally Bayesian Incentive Compatible Stable Matching," Working Paper, Concordia University.

Majumdar, D. And A. Sen (2004): "Ordinally Bayesian Incentive Compatible Voting Rules," Econometrica, 72, 523-540.

Manelli, A. M. And D. R. Vincent (2010): "Bayesian and Dominant Strategy Implementation in the Independent Private Values Model," Econometrica, 78, 1905-1938.

Manjunath, V. (2014): "Efficient and strategy-proof social choice when preferences are single-dipped," International Journal of Game Theory, 43, 579-597.

Mishra, D., A. Pramanik, And S. Roy (2015): "Local Incentive Compatibility with Transfers," Working Paper, Indian Statistical Institute.

Moulin, H. (1980): "On Strategyproofness and Single-peakedness," Public Choice, 35, 437455.

(1983): The Strategy of Social Choice, North-Holland.

Muto, N. And S. SATo (2014): "A decomposition of strategy-proofness," Working Paper, Fukuoka University.

Peremans, W. And T. Storcken (1999): "Strategy-proofness on single-dipped preference domains," in Proceedings of the International Conference, Logic, Game Theory and Social Choice, 296-313.

REFFGen, A. (2015): "Strategy-proof social choice on multiple and multi-dimensional singlepeaked domains," Journal of Economic Theory, 157, 349-383.

SAPORITI, A. (2009): "Strategy-proofness and single-crossing," Theoretical Economics, 4, $127-163$.

SATO, S. (2013): "A sufficient condition for the equivalence of strategy-proofness and nonmanipulability by preferences adjacent to the sincere one," Journal of Economic Theory, $148,259-278$.

Satterthwaite, M. (1975): "Strategy-proofness and Arrow's Conditions: Existence and Correspondence Theorems for Voting Procedures and Social Welfare Functions," Journal of Economic Theory, 10, 187-217. 
Weymark, J. A. (2008): "Strategy-Proofness and the Tops-Only Property," Journal of Public Economic Theory, 10, 7-26. 